\title{
EFFECT OF SHORTAGES ON LIMITED STORAGE INVENTORY SYSTEM FOR DECAYING ITEMS WITH EXPONENTIAL DEMAND
}

\author{
Dr. Pankaj Agarwal \\ Information Technology \\ Institute of Management Studies Noida,U.P.,India
}

\begin{abstract}
In this paper a two-warehouse inventory model for deteriorating items with exponential demand rate is developed and analysed. In the present model shortages are allowed and completely backlogged. It is assumed that the owned warehouse has a fixed capacity whether the rented warehouse (RW) has unlimited capacity. Effect of inflation is taken in consideration.
\end{abstract}

Keywords: Demand, Inventory, Inflation, Shortages Etc.

\section{INTRODUCTION}

The important problem associated with the inventory maintenance is to decide where to stock the goods. This problem does not have attracted the attention of researchers. In the existing literature, it is found that classical inventory models generally deal with a single storage facility. The basic assumption in these models is that the management has owned storage with unlimited capacity. In the field of inventory management, the unlimited capacity of storage is not true. When an attractive price discount for bulk purchase is available or the cost of procuring goods is higher than the other inventory related costs or there are some problems in frequent procurement or the demand of items is very high, management then decides to purchase (or produce) a huge quantity of items at a time. These items can not be stored in the existing storage, viz the owned warehouse (OW) with limited capacity. Then for storing the excess of items, a warehouse is hired on a rental basis. This rented warehouse (RW) may be located near the OW or a little away from it. It is generally assumed that, holding cost in the RW is greater than the same as in OW. Hence, the items are stored first in $\mathrm{OW}$ and only excess stock is stored in RW. Further the items of RW are transferred to OW in a continuous release pattern to meet the demand until the stock level in the RW is emptied and then the items of OW are released. A two warehouse inventory model was discussed by Hartely [1976]. Sarma [1983] developed a deterministic inventory model with infinite production rate and two levels of storage. Dave [1988] rectified the errors and gave a complete solution of the model given by Sarma [1983]. In the above models, the analysis is carried out without taking shortages. Goswami and Chaudhuri [1992] considered two storage models with and without shortages, allowing time dependent demand. Bhunia and Maiti [1994] developed the same inventory models correcting and modifying the assumption of Goswami and Chaudhuri [1992]. Also, by considering constant demand, Sarma [1987] presented a model for deteriorating items with an infinite replenishment rate allowing for shortages. Bhunia and Maiti [1997] studied a two warehouse inventory model for deteriorating items considering linearly time dependent demand and shortages. Buzacott and Misra [1975] simultaneously developed EOQ models with constant demand and a single inflation rate for all associated costs. Bierman and Thomas [1977] then proposed an inflation model for the EOQ in which the time value of money is also incorporated. Bose et al. [1995] developed an EOQ inventory model under inflation and time discounting. Recently, Yang et al. [2006] generalized the inventory model under inflation for variable demand.

In the last few years, inventory problems involving time variable demand pattern have received attention from several researchers. This type of problem was first discussed by Stanfel and Sivazlian [1975]. Next, Silver and Meal [1973] established an approximate solution technique of a deterministic inventory model with time dependent demand. Donaldson [1977] developed an optimal algorithm for solving the 


\section{International Journal of Engineering Applied Sciences and Technology, 2019 \\ Vol. 4, Issue 3, ISSN No. 2455-2143, Pages 438-453 \\ Published Online July 2019 in IJEAST (http://www.ijeast.com)}

classical no-shortage inventory model analytically with linear trend in demand over a fixed time horizon. However, the solution procedure requires a lot of computational work and can not be easily employed to determine the values of the optimal decision variable. To remove the computational and conceptual complexity of Donaldson's [1977] optimal analytic approach, several researchers employed search methods for solving the problem. Among them, Silver [1979], Henry [1979], Phelps [1980], Buchanan [1980], Mitra et al. [1984], Ritchie [1984] and others are worth mentioning, but none of them considered shortages. Considering shortages, Dave [1989], Deb and Chaudhari [1987], Goyal et. al. [1992], Dutta and Pal [1992], and Horiga [1993, 1994] developed a solution following Donaldson's or alternative approaches. In these papers, the solution procedure was computationally complicated except Dutta and Pal where it was very simple and easy to calculate the values of decision variables. Other papers of related topic were written by Chung and Ting [1993], Goyal et al. [1996], Bhunia and Maiti [1999], Chakravarti and Chaudhari [1997] and others.

\section{ASSUMPTIONS}

The mathematical model of the two-warehouse inventory problems is based on the following assumptions:

1. Shortages are allowed and completely backlogged.

2. Lead time is zero and the initial inventory level is zero.

3. The owned warehouse (OW) has a fixed capacity of $\mathrm{W}$ units.

4. The rented warehouse (RW) has unlimited capacity.

5. The inventory costs (including holding cost and deterioration

cost) in RW are higher than those in OW.

In addition the following notations are used throughout this paper.

W The capacity of owned warehouse (OW).

$a e^{b t} \quad$ The demand rate at time $t$, we assume that $\mathrm{e}^{\mathrm{bt}}$ is deterministic exponential rate of $D$ units per unit time.

$\alpha \quad$ The deterioration rate in OW, where $0<\alpha<1$.

$\beta$ The deterioration rate in $\mathrm{RW}$, where $0<\beta<1$ and $\beta>\alpha$.

$r \quad$ The inflation rate

$\mathrm{C}_{\mathrm{p}}$ The purchasing cost per unit.

$\mathrm{c}_{0} \quad$ The replenishment cost per order. $\mathrm{ch}_{1}$ The holding cost per unit per unit time in $\mathrm{OW}$.

$\mathrm{ch}_{2}$ The holding cost per unit per unit time in $\mathrm{RW}$.

$\mathrm{C}_{\mathrm{s}} \quad$ The shortage cost per unit time.

$\mathrm{Tc}_{\mathrm{i}}$ The present value of the total relevant cost per unit time for model $\mathbf{i}, \mathbf{i}=1,2$.

$\mathrm{I}_{0}(\mathrm{t})$ The inventory level in OW at time $\mathrm{t}$.

$I_{r}(t)$ The inventory level in $\mathrm{RW}$ at time $\mathrm{t}$.

$\mathrm{S}(\mathrm{t})$ The shortage level at time $\mathrm{t}$.

$t_{0} \quad$ The time at which the inventory level reaches to zero in $\mathrm{OW}$.

$t_{r} \quad$ The time at which the inventory level reaches to zero in $\mathrm{RW}$.

$t_{s} \quad$ The time at which the shortages level reaches to the lowest point in the replenishment cycle.

\section{Mathematical Formulation and Analysis}

There is only one storage model (i.e. the traditional model) associated with this two warehouses inventory problem found in the previous literature. The traditional model is depicted graphically in fig 1. It starts with an instant replenishment and ends with shortages. In the other model the demand will be met at the end of each replenishment cycle, that is, the inventory level start with shortages and ends without shortages. The proposed inventory model is depicted graphically in fig 2 .

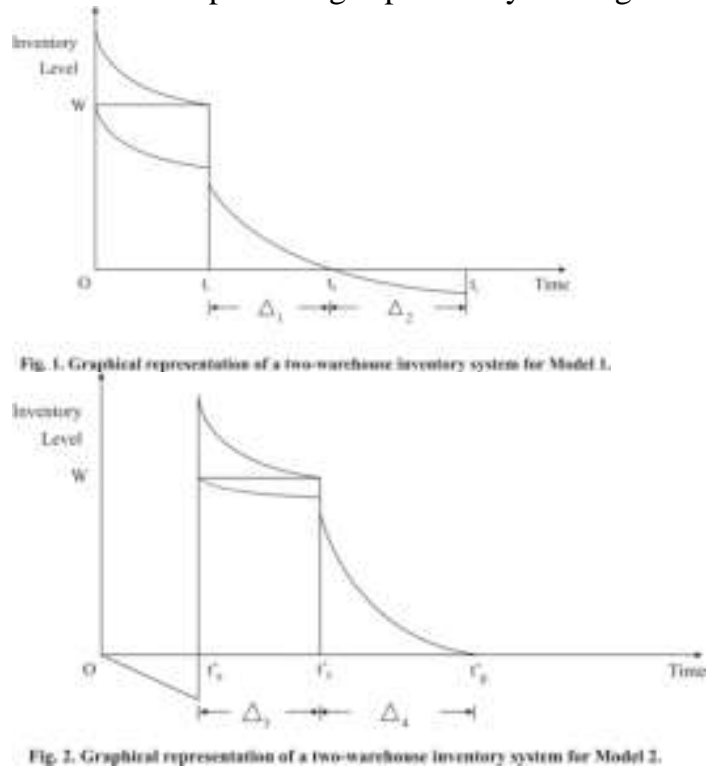

There are two possible shortages models under the assumption described above. These two models are depicted graphically in figs 1 and 2. The traditional model (i.e. model 1), it 
starts with an instant order and ends with shortages.

For the traditional model, at time $\mathrm{t}=0$, a lot size of certain units enters the system, from which a portion is backlogged towards previous shortages. W units are kept in OW and the rest is stored in $\mathrm{RW}$. The goods of $\mathrm{OW}$ are consumed only after consuming the goods kept in $\mathrm{RW}$. During the interval $\left(0, \mathrm{t}_{\mathrm{r}}\right)$, the inventory in RW gradually decreases due to demand and deterioration and it vanishes at $\mathrm{t}=\mathrm{t}_{\mathrm{r}}$. In $\mathrm{OW}$, the inventory $\mathrm{W}$ decreases during $\left(0, \mathrm{t}_{\mathrm{r}}\right)$ due to deterioration only, but during $\left(t_{r}, t_{0}\right)$ the inventory is depleted due to both demand and deterioration. By the time $t_{0}$, both warehouses become empty and thereafter the shortages are allowed to occur. The shortage quantity is supplied to customers at the beginning of the next cycle. By the time $t_{s}$, the replenishment cycle restarts. The objective of the traditional model is to determine the timings of $t_{r}$ and $t_{s}-t_{0}$ so that the total relevant cost (including holding, deterioration, shortage and ordering costs) per unit time of the inventory system is minimum.

For model $I$ during the interval $\left(0, t_{r}\right)$, the inventory level at time $\mathrm{t}$ in $\mathrm{RW}$ and $\mathrm{OW}$ is governed by the following differential equation:

$$
\begin{aligned}
& \frac{\mathrm{dI}_{\mathrm{r}}(\mathrm{t})}{\mathrm{dt}}=-a \mathrm{e}^{\mathrm{bt}}-\beta \mathrm{I}_{\mathrm{r}}(\mathrm{t}), \\
& 0 \leq \mathrm{t} \leq \mathrm{t}_{\mathrm{r}}
\end{aligned}
$$

with the boundary condition $I_{r}\left(t_{r}\right)=0$ and

$$
\begin{aligned}
& \frac{\mathrm{dI}_{0}(\mathrm{t})}{\mathrm{dt}}=-\alpha \mathrm{I}_{0}(\mathrm{t}), \\
& 0 \leq \mathrm{t} \leq \mathrm{t}_{\mathrm{r}}
\end{aligned}
$$

with the initial condition $\mathrm{I}_{0}(0)=\mathrm{W}$, respectively. While during the interval $\left(t_{r}, t_{0}\right)$ , the inventory level at $\mathrm{OW}, \mathrm{I}_{0}(\mathrm{t})$, is governed by the following differential equation

$$
\begin{aligned}
& \frac{\mathrm{dI}_{0}(\mathrm{t})}{\mathrm{dt}}=-\mathrm{ae}^{\mathrm{bt}}-\alpha \mathrm{I}_{0}(\mathrm{t}), \\
& \mathrm{t}_{\mathrm{r}} \leq \mathrm{t} \leq \mathrm{t}_{0}
\end{aligned}
$$

with the boundary condition $\mathrm{I}_{0}\left(\mathrm{t}_{0}\right)=0$ . Similarly, during $\left(\mathrm{t}_{0}, \mathrm{t}_{\mathrm{s}}\right)$, the shortage level at time $\mathrm{t}, \mathrm{S}(\mathrm{t})$ is governed by the following differential equation :

$$
\begin{aligned}
& \frac{\mathrm{dS}(\mathrm{t})}{\mathrm{dt}}=\mathrm{ae}^{\mathrm{bt}}, \\
& \mathrm{t}_{0} \leq \mathrm{t} \leq \mathrm{t}_{\mathrm{s}}
\end{aligned}
$$

With the boundary condition $\mathrm{S}\left(\mathrm{t}_{0}\right)=0$.

The solution of (1) is as follows

$$
\begin{array}{ll} 
& \mathrm{I}_{\mathrm{r}}\left(\mathrm{t}_{\mathrm{r}}\right) \mathrm{e}^{\beta \mathrm{t}_{\mathrm{r}}}=-\int a e^{(\mathrm{b}+\beta) \mathrm{t}_{\mathrm{r}}} d \mathrm{t}_{\mathrm{r}}+C \\
\because \quad & \mathrm{I}_{\mathrm{r}}\left(\mathrm{t}_{\mathrm{r}}\right)=0 \\
\mathrm{I}_{\mathrm{r}}(\mathrm{t})= & \mathrm{e}^{-\beta \mathrm{t}} \int_{\mathrm{t}}^{\mathrm{t}_{\mathrm{r}}} a \mathrm{e}^{(\mathrm{b}+\beta) \mathrm{u}} \mathrm{du}
\end{array}
$$

The solution of (2) is as follows

$$
\begin{aligned}
& \mathrm{I}_{0}(\mathrm{t})=\mathrm{We}^{-\alpha \mathrm{t}}, \\
& 0 \leq \mathrm{t} \leq \mathrm{t}_{\mathrm{r}}
\end{aligned}
$$

The solution of (3) is as follows

$$
\begin{gathered}
\mathrm{I}_{0}(\mathrm{t}) \mathrm{e}^{\alpha \mathrm{t}}=\int \mathrm{ae}^{(\mathrm{b}+\alpha) \mathrm{t}_{0}} \mathrm{dt}_{0}-\int \mathrm{ae}^{(\mathrm{b}+\alpha) \mathrm{t}} \mathrm{dt} \\
\mathrm{I}_{0}(\mathrm{t})=\mathrm{e}^{-\alpha \mathrm{t}} \int_{\mathrm{t}}^{\mathrm{t}_{0}} \mathrm{ae}^{(\mathrm{b}+\alpha) \mathrm{u}} \mathrm{du}, \\
\mathrm{t}_{\mathrm{r}} \leq \mathrm{t} \leq \mathrm{t}_{0}
\end{gathered}
$$

The solution of (4) is as follows

$$
\begin{aligned}
& S(t)=\int a e^{b t} d t-\int a e^{b t_{0}} d t_{0} \\
& S(t)=\int_{t_{0}}^{t} a e^{b u} d u,
\end{aligned}
$$$$
\mathrm{t}_{0} \leq \mathrm{t} \leq \mathrm{t}_{\mathrm{s}}
$$

Using the condition for $\mathrm{I}_{0}(\mathrm{t})$ at $\mathrm{t}=\mathrm{t}_{\mathrm{r}}$ , we have from (6) and (7), that

$$
\mathrm{I}_{0}(\mathrm{t})=\mathrm{We}^{-\alpha \mathrm{t}}
$$

and

$$
I_{0}(t)=e^{-\alpha t} \int_{t}^{t_{0}} a e^{(b+\alpha) u} d u
$$

or

$$
I_{0}\left(t_{r}\right)=W e^{-\alpha t_{r}}=e^{-\alpha t_{r}} \int_{t_{r}}^{t_{0}} a e^{(b+\alpha) u} d u
$$

which implies that 


$$
\mathrm{W}=\int_{\mathrm{t}_{\mathrm{r}}}^{\mathrm{t}_{0}} \mathrm{a} \mathrm{e}^{(\mathrm{b}+\alpha) \mathrm{t}} \mathrm{dt}
$$

$$
\mathrm{W}=\frac{\mathrm{a}}{(\mathrm{b}+\alpha)}\left[\mathrm{e}^{(\mathrm{b}+\alpha) \mathrm{t}_{0}}-\mathrm{e}^{(\mathrm{b}+\alpha) \mathrm{t}_{\mathrm{r}}}\right]
$$

$\frac{(b+\alpha) W}{a}=e^{(b+\alpha) t_{r}}\left[e^{(b+\alpha) t_{0}-(b+\alpha) t_{r}}-1\right]$

$\mathrm{t}_{0}=\mathrm{t}_{\mathrm{r}}+\ln \left[1+\frac{(\mathrm{b}+\alpha)}{\mathrm{a}} \mathrm{We}^{-(\mathrm{b}+\alpha) \mathrm{t}_{\mathrm{r}}}\right] /(\mathrm{b}+\alpha)$

Note that from (11) $t_{0}$ is a function of $t_{r}$, therefore $t_{0}$ is not a decision variable in model I. Thus, the cumulative inventories in $\mathrm{RW}$ during $\left(0, \mathrm{t}_{\mathrm{r}}\right)$ and $\mathrm{OW}$ during $\left(0, \mathrm{t}_{0}\right)$ are

$$
\int_{0}^{t_{\mathrm{r}}} I_{r}(t) d t=\int_{0}^{t_{r}} e^{-\beta t} \int_{t}^{t_{r}} a e^{(b+\beta) u} d u d t
$$

and $\quad \int_{0}^{t_{0}} I_{0}(t) d t=\int_{0}^{t_{r}} I_{0}(t) d t+\int_{t_{r}}^{t_{0}} I_{0}(t) d t$

$\int_{0}^{t_{0}} I_{0}(t) d t=\int_{0}^{t_{r}} W e^{-\alpha t} d t+\int_{t_{r}}^{t_{0}} e^{-\alpha t} \int_{t}^{t_{0}} e^{(\alpha+b) u} d u d t$ ... (13)

respectively and the cumulative shortages during $\left(\mathrm{t}_{0}, \mathrm{t}_{\mathrm{s}}\right)$ is

$$
\int_{\mathrm{t}_{0}}^{\mathrm{t}_{\mathrm{s}}} \int_{\mathrm{t}_{0}}^{\mathrm{t}} \mathrm{ae}^{\mathrm{bu}} \mathrm{dudt} \text {. }
$$

As a result the present value of the inventory holding cost in RW and $\mathrm{OW}$ are

$$
\begin{aligned}
& \operatorname{ch}_{2} \int_{0}^{t_{\mathrm{r}}} e^{-r t} I_{r}(t) d t \\
& \quad=\operatorname{ch}_{2} \int_{0}^{t_{\mathrm{r}}} e^{-(\beta+r) t} \int_{\mathrm{t}}^{t_{\mathrm{r}}} a e^{(b+\beta) u} d u d t \\
& =\operatorname{ch}_{2} \int_{0}^{t_{\mathrm{r}}} a e^{(b+\beta) u}\left(\int_{0}^{\mathrm{u}} e^{-(\beta+r) t} d t\right) d u
\end{aligned}
$$

$$
\begin{aligned}
& \frac{\mathrm{ch}_{2}}{(\beta+\mathrm{r})} \int_{0}^{\mathrm{t}_{\mathrm{r}}} \mathrm{a} \mathrm{e}^{(\mathrm{b}+\beta) \mathrm{u}}\left(1-\mathrm{e}^{-(\beta+\mathrm{r}) \mathrm{u}}\right) \mathrm{du} \\
& =\frac{\mathrm{ch}}{(\beta+\mathrm{r})} \int_{0}^{\mathrm{t}_{\mathrm{r}}} \mathrm{a}\left(\mathrm{e}^{(\mathrm{b}+\beta) \mathrm{t}}-\mathrm{e}^{(\mathrm{b}-\mathrm{r}) \mathrm{t}}\right) \mathrm{dt}
\end{aligned}
$$

and

$$
\begin{aligned}
& \operatorname{ch}_{1} \int_{0}^{t_{0}} e^{-r t} I_{0}(t) d t \\
& =\operatorname{ch}_{1}\left[\int_{0}^{t_{\mathrm{r}}} e^{-r t} I_{0}(t) d t+\int_{t_{r}}^{t_{0}} e^{-r t} I_{0}(t) d t\right]
\end{aligned}
$$$$
=\operatorname{ch}_{1}\left[\int_{0}^{t_{\mathrm{r}}} \mathrm{We}^{-(\alpha+r) t} \mathrm{dt}+\int_{\mathrm{t}_{\mathrm{r}}}^{\mathrm{t}_{0}} \mathrm{e}^{-(\alpha+r) t} \int_{\mathrm{t}}^{\mathrm{t}_{0}} \mathrm{a} \mathrm{e}^{(\mathrm{b}+\alpha) \mathrm{u}} \mathrm{dudt}\right]
$$$$
=\operatorname{ch}_{1}\left[\int_{0}^{t_{\mathrm{r}}} \mathrm{e}^{-(\alpha+r) t} \int_{\mathrm{t}_{\mathrm{r}}}^{\mathrm{t}_{0}} a e^{(b+\alpha) u} d u d t+\int_{\mathrm{t}_{\mathrm{r}}}^{\mathrm{t}_{0}} \mathrm{e}^{-(\alpha+r) t} \int_{\mathrm{t}}^{\mathrm{t}_{0}} a e^{(b+\alpha) u} d u d t\right]
$$$$
=\operatorname{ch}_{1}\left[\int_{t_{r}}^{t_{0}} a e^{(b+\alpha) u}\left(\int_{0}^{t_{r}} e^{-(\alpha+r) t} d t\right) d u+\int_{t_{r}}^{t_{0}} a e^{(b+\alpha) u}\left(\int_{t_{r}}^{u} e^{-(\alpha+r) t} d t\right) d u\right]
$$$$
=\operatorname{ch}_{1}\left[\int_{t_{\mathrm{r}}}^{\mathrm{t}_{0}} \frac{\mathrm{a}}{\alpha+\mathrm{r}} \mathrm{e}^{(\mathrm{b}+\alpha) \mathrm{u}}\left(1-\mathrm{e}^{-(\alpha+\mathrm{r}) \mathrm{t}_{\mathrm{r}}}\right) \mathrm{du}\right.
$$

$$
\begin{array}{r}
+\int_{\mathrm{t}_{\mathrm{r}}}^{\mathrm{t}_{0}} \frac{\mathrm{a}}{\alpha+\mathrm{r}} \mathrm{e}^{(\mathrm{b}+\alpha) \mathrm{u}}\left(\mathrm{e}^{-(\alpha+\mathrm{r}) \mathrm{t}_{\mathrm{r}}}-\mathrm{e}^{-(\alpha+\mathrm{r}) \mathrm{u}}\right) \mathrm{du} \\
=\frac{\mathrm{ch}}{\alpha+\mathrm{r}} \int_{\mathrm{t}_{\mathrm{r}}}^{\mathrm{t}_{0}} \mathrm{a}\left(\mathrm{e}^{(\mathrm{b}+\alpha) \mathrm{u}}-\mathrm{e}^{(\mathrm{b}-\mathrm{r}) \mathrm{u}}\right) \mathrm{du} \\
=\frac{\mathrm{ch}_{1}}{\alpha+\mathrm{r}} \int_{\mathrm{t}_{\mathrm{r}}}^{\mathrm{t}_{0}} \mathrm{a}\left(\mathrm{e}^{(\mathrm{b}+\alpha) \mathrm{t}}-\mathrm{e}^{(\mathrm{b}-\mathrm{r}) \mathrm{t}}\right) \mathrm{dt}
\end{array}
$$


Respectively and the present value of the shortage cost is

$$
\begin{aligned}
& c_{s} \int_{t_{0}}^{t_{s}} e^{-r t} \int_{t_{0}}^{t} a e^{b u} d u d t \\
= & c_{s} \int_{t_{0}}^{t_{s}} a e^{b u}\left(\int_{u}^{t_{s}} e^{-r t} d t\right) d u \\
= & \frac{c_{s}}{r} \int_{t_{0}}^{t_{s}} a e^{b t}\left(e^{-r t}-e^{-r t_{s}}\right) d t \\
= & \frac{c_{s}}{r} \int_{t_{0}}^{t_{s}} a\left(e^{(b-r) t}-e^{b t-r t_{s}}\right) d t
\end{aligned}
$$

In addition, the amounts of deteriorated items in both RW and OW during $\left(0, \mathrm{t}_{0}\right)$ are $\beta \int_{0}^{t_{\mathrm{r}}} \mathrm{I}_{\mathrm{r}}(\mathrm{t}) \mathrm{dt}$ and $\alpha \int_{0}^{\mathrm{t}_{0}} \mathrm{I}_{0}(\mathrm{t}) \mathrm{dt}$. Therefore, the present value of the cost for the deteriorated items is

$$
\begin{gathered}
\mathrm{c}_{\mathrm{p}}\left[\beta \int_{0}^{\mathrm{t}_{\mathrm{r}}} \mathrm{e}^{-\mathrm{rt}} \mathrm{I}_{\mathrm{r}}(\mathrm{t}) \mathrm{dt}+\alpha \int_{0}^{\mathrm{t}_{0}} \mathrm{e}^{-\mathrm{rt}} \mathrm{I}_{0}(\mathrm{t}) \mathrm{dt}\right] \\
=c_{p}\left[\beta \int_{0}^{t_{r}} e^{-(\beta+r) t} \int_{t}^{t_{r}} a e^{(b+\beta) u} d u d t+\alpha\left\{\int_{0}^{t_{\mathrm{r}}} e^{-(\alpha+r) t} \int_{t_{r}}^{t_{0}} a e^{(b+\beta) u} d u d t\right.\right. \\
\left.\left.+\int_{\mathrm{t}_{\mathrm{r}}}^{\mathrm{t}_{0}} \mathrm{e}^{-(\alpha+\mathrm{r}) \mathrm{t}} \int_{\mathrm{t}}^{\mathrm{t}_{0}} \mathrm{ae} \mathrm{e}^{(\mathrm{b}+\alpha) \mathrm{u}} \mathrm{dudt}\right\}\right] \\
=c_{\mathrm{p}}\left[\frac{\beta}{\beta+\mathrm{r}} \int_{0}^{\mathrm{t}_{\mathrm{r}}} \mathrm{ae} \mathrm{e}^{(\mathrm{b}+\beta) \mathrm{u}\left(1-\mathrm{e}^{-(\beta+\mathrm{r}) \mathrm{u}}\right) \mathrm{du}+\frac{\alpha}{\alpha+\mathrm{r}}\left\{\int_{\mathrm{t}_{\mathrm{r}}}^{\mathrm{t}_{0}} \mathrm{ae} \mathrm{e}^{(\mathrm{b}+\alpha) \mathrm{u}}\left(1-\mathrm{e}^{-(\alpha+\mathrm{r}) \mathrm{t}_{\mathrm{r}}}\right) \mathrm{du}\right.}\right. \\
\left.+\int_{\mathrm{t}_{\mathrm{r}}}^{\mathrm{t}_{0}} \mathrm{ae} \mathrm{e}^{(\mathrm{b}+\alpha) \mathrm{u}}\left(\mathrm{e}^{-(\alpha+\mathrm{r}) \mathrm{t}_{\mathrm{r}}}-\mathrm{e}^{-(\alpha+\mathrm{r}) \mathrm{u}}\right) \mathrm{du}\right\}
\end{gathered}
$$

$$
\begin{aligned}
& =c_{p}\left[\frac{\beta}{\beta+r} \int_{0}^{t_{r}} a\left(e^{(b+\beta) u}-e^{(b-r) u}\right) d u+\frac{\alpha}{\alpha+r} \int_{t_{r}}^{t_{0}} e^{(b+\alpha) u}\left(1-e^{-(\alpha+r) u}\right) d u\right] \\
& =c_{p}\left[\frac{\beta}{\beta+r} \int_{0}^{t_{r}} a\left(e^{(b+\beta) t}-e^{(b-r) t}\right) d t+\frac{\alpha}{\alpha+r} \int_{t_{r}}^{t_{0}} a\left(e^{(b+\alpha) t}-e^{(b-r) t}\right) d t\right]
\end{aligned}
$$

Consequently, the present value of the total relevant cost per unit time for model I during the cycle $\left(0, t_{s}\right)$ is

$$
\begin{gathered}
\mathrm{Tc}_{1}=\left\{\mathrm{c}_{0}+\frac{\mathrm{ch}_{2}}{\beta+\mathrm{r}} \int_{0}^{\mathrm{t}_{\mathrm{r}}} \mathrm{a}\left(\mathrm{e}^{(\mathrm{b}+\beta) \mathrm{t}}-\mathrm{e}^{(\mathrm{b}-\mathrm{r}) \mathrm{t}}\right) \mathrm{dt}+\frac{\mathrm{ch}_{1}}{\alpha+\mathrm{r}} \int_{\mathrm{t}_{\mathrm{r}}}^{\mathrm{t}_{0}} \mathrm{a}\left(\mathrm{e}^{(\mathrm{b}+\alpha) \mathrm{t}}-\mathrm{e}^{(\mathrm{b}-\mathrm{r}) \mathrm{t}}\right) \mathrm{dt}\right. \\
+\frac{\mathrm{c}_{\mathrm{s}}}{\mathrm{r}} \int_{\mathrm{t}_{0}}^{\mathrm{t}_{\mathrm{s}}} \mathrm{a}\left(\mathrm{e}^{(\mathrm{b}-\mathrm{r}) \mathrm{t}}-\mathrm{e}^{\mathrm{bt}-\mathrm{rt}_{\mathrm{s}}}\right) \mathrm{dt}
\end{gathered}
$$$$
+c_{p}\left[\frac{\beta}{\beta+r} \int_{0}^{t_{r}} a\left(e^{(b+\beta) t}-e^{(b-r) t}\right) d t\right.
$$$$
\left.\left.+\frac{\alpha}{\alpha+r} \int_{\mathrm{t}_{\mathrm{r}}}^{\mathrm{t}_{0}} \mathrm{a}\left(\mathrm{e}^{(\mathrm{b}+\alpha) \mathrm{t}}-\mathrm{e}^{(\mathrm{b}-\mathrm{r}) \mathrm{t}}\right) \mathrm{dt}\right]\right\} / \mathrm{t}_{\mathrm{s}}
$$$$
=\left\{c_{0}+\frac{c h_{2}+\beta c_{p}}{\beta+r}\left[\frac{1}{b+\beta}\left(e^{(b+\beta) t}-1\right)-\frac{1}{r-b}\left(1-e^{(b-r) t}\right)\right]_{0}^{t_{r}} a\right.
$$$$
+\frac{\mathrm{ch}_{1}+\alpha c_{p}}{\alpha+r}\left[\frac{1}{b+\alpha} e^{(b+\alpha) t}-\frac{1}{b-r} e^{(b-r) t}\right]_{t_{r}}^{t_{0}} a
$$$$
+\frac{c_{s}}{r}\left[\frac{e^{(b-r) t}}{b-r}+\frac{e^{b t-r t_{s}}}{b}\right]_{t_{0}}^{t_{s}} a / t_{s}
$$

$$
=\left\{c_{0}+\frac{\mathrm{ch}_{2}+\beta c_{p}}{\beta+r}\left[\frac{1}{b+\beta}\left(e^{(b+\beta) t_{r}}-1\right)-\frac{1}{r-b}\left(1-e^{(b-r) t_{r}}\right)\right] a\right.
$$


$+\frac{\mathrm{ch}_{1}+\alpha \mathrm{c}_{\mathrm{p}}}{\alpha+\mathrm{r}}\left[\frac{\mathrm{e}^{(\mathrm{b}+\alpha) \mathrm{t}_{0}}}{\mathrm{~b}+\alpha}-\frac{\mathrm{e}^{(\mathrm{b}+\alpha) \mathrm{t}_{\mathrm{r}}}}{\mathrm{b}+\alpha}+\frac{\mathrm{e}^{(\mathrm{b}-\mathrm{r}) \mathrm{t}_{0}}}{\mathrm{r}-\mathrm{b}}-\frac{\mathrm{e}^{(\mathrm{b}-\mathrm{r}) \mathrm{t}_{\mathrm{r}}}}{\mathrm{r}-\mathrm{b}}\right] \mathrm{a}$

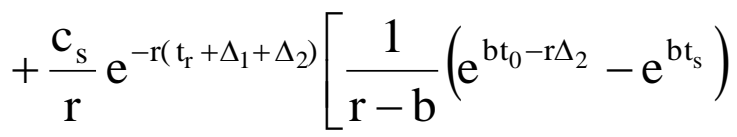

$\left.+\frac{c_{s}}{r}\left[-\frac{e^{(b-r) t_{s}}}{r-b}+\frac{e^{(b-r) t_{0}}}{r-b}-\frac{e^{(b-r) t_{s}}}{b}+\frac{e^{b t_{0}-r t_{s}}}{b}\right] a\right\} / t_{s}$

$\left.\left.-\frac{1}{\mathrm{~b}}\left(\mathrm{bt}_{\mathrm{s}}-\mathrm{bt}_{0}\right)\right]\right\} /\left(\mathrm{t}_{\mathrm{r}}+\Delta_{1}+\Delta_{2}\right)$

$=\left\{c_{0}+\frac{c_{2}+\beta c_{p}}{\beta+r}\left[\frac{1}{b+\beta}\left(e^{(b+\beta) t_{r}}-1\right)-\frac{1}{r-b}\left(1-e^{(b-r) t_{r}}\right)\right] a\right.$

$=\left\{c_{0}+\frac{\mathrm{ch}_{2}+\beta \mathrm{c}_{\mathrm{p}}}{\beta+\mathrm{r}}\left[\frac{1}{\mathrm{~b}+\beta}\left(\mathrm{e}^{(\mathrm{b}+\beta) \mathrm{t}_{\mathrm{r}}}-1\right)-\frac{1}{\mathrm{r}-\mathrm{b}}\left(1-\mathrm{e}^{(\mathrm{b}-\mathrm{r}) \mathrm{t}_{\mathrm{r}}}\right)\right] \mathrm{a}\right.$

$+\frac{\mathrm{ch}_{1}+\alpha \mathrm{c}_{\mathrm{p}}}{\alpha+\mathrm{r}}\left[\mathrm{W}-\frac{\mathrm{a}}{\mathrm{r}-\mathrm{b}} \mathrm{e}^{-\mathrm{rt}_{\mathrm{r}}}\left(\mathrm{e}^{\mathrm{bt_{ \textrm {r } }}}-\mathrm{e}^{\mathrm{bt} \mathrm{t}_{0}-\mathrm{rt}_{0}+\mathrm{rt}_{\mathrm{r}}}\right)\right]$

$+\frac{\mathrm{ch}_{1}+\alpha \mathrm{c}_{\mathrm{p}}}{\alpha+\mathrm{r}}\left[\mathrm{W}-\frac{\mathrm{a}}{\mathrm{r}-\mathrm{b}} \mathrm{e}^{-(\mathrm{r}-\mathrm{b}) \mathrm{t}_{\mathrm{r}}}\left(1-\mathrm{e}^{(\mathrm{b}-\mathrm{r}) \Delta_{1}}\right)\right]$

$\left.+\frac{\mathrm{c}_{\mathrm{s}}}{\mathrm{r}} \mathrm{e}^{-\mathrm{r}\left(\mathrm{t}_{\mathrm{r}}+\Delta_{1}+\Delta_{2}\right)}\left[\frac{1}{\mathrm{r}-\mathrm{b}}\left(\mathrm{e}^{\mathrm{bt}_{0}-\mathrm{r} \Delta_{2}}-\mathrm{e}^{\mathrm{bt}}\right)-\Delta_{2}\right] \mathrm{a}\right\} /\left(\mathrm{t}_{\mathrm{r}}+\Delta_{1}+\Delta_{2}\right)$

$\left.+\frac{c_{s}}{r} e^{-r t_{s}}\left[-\frac{e^{b t_{s}}}{r-b}+\frac{e^{b t_{0}-r t_{0}+r t_{s}}}{r-b}-\frac{e^{b t_{s}}}{b}+\frac{e^{b t_{0}}}{b}\right]\right\} / t_{s}$

where

$\Delta_{1}=\mathbf{t}_{\mathrm{o}}-\mathbf{t}_{\mathrm{r}}=\operatorname{In}\left[1+\frac{(\mathrm{b}+\alpha) \mathrm{W}}{\mathrm{a}} \mathrm{e}^{-(\mathrm{b}+\alpha) \mathbf{t}_{\mathrm{r}}}\right] /(\mathrm{b}+\alpha)$

$=\left\{c_{0}+\frac{\mathrm{ch}_{2}+\beta \mathrm{c}_{\mathrm{p}}}{\beta+\mathrm{r}}\left[\frac{1}{\mathrm{~b}+\beta}\left(\mathrm{e}^{(\mathrm{b}+\beta) \mathrm{t}_{\mathrm{r}}}-1\right)-\frac{1}{\mathrm{r}-\mathrm{b}}\left(1-\mathrm{e}^{(\mathrm{b}-\mathrm{r}) \mathrm{t}_{\mathrm{r}}}\right)\right] \mathrm{a}\right.$ and

$\Delta_{2}=\mathrm{t}_{\mathrm{s}}-\mathrm{t}_{0}$

To distinguish easily, the notations $\mathrm{t}_{0}$, $t_{r}, t_{s}$ are replaced by $t_{0}^{\prime}, t_{r}^{\prime}, t_{s}^{\prime}$ in model 2. for $+\frac{c h_{1}+\alpha c_{p}}{\alpha+r}\left[W-\frac{a}{r-b} e^{-r t_{r}}\left(e^{b t_{r}}-e^{b t_{0}-r\left(t_{0}-t_{r}\right)}\right)\right]$ model 2 , the present value of ordering cost is $\mathrm{C}_{0} \mathrm{e}^{-\mathrm{rt}_{\mathrm{s}}^{\prime}}$. By using a similar argument as in model 1, the present value of the total relevant cost per unit time for model 2 is obtained as follows :

$\left.+\frac{\mathrm{c}_{\mathrm{s}}}{\mathrm{r}} \mathrm{e}^{-\mathrm{rt}}\left[\frac{1}{\mathrm{r}-\mathrm{b}}\left(\mathrm{e}^{\mathrm{bt} \mathrm{t}_{0}-\mathrm{r}\left(\mathrm{t}_{0}-\mathrm{t}_{\mathrm{s}}\right)}-\mathrm{e}^{\mathrm{bt_{ \textrm {s } }}}\right)-\frac{1}{\mathrm{~b}}\left(\mathrm{e}^{\mathrm{bt_{ \textrm {s } }}}-\mathrm{e}^{\mathrm{bt} \mathrm{t}_{0}}\right)\right]\right\} / \mathrm{t}_{\mathrm{s}}$ $\mathrm{Tc}_{2}=\mathrm{e}^{-\mathrm{rt} \mathrm{s}_{\mathrm{s}}^{\prime}}\left\{\mathrm{c}_{0}+\frac{\mathrm{c}_{\mathrm{s}}}{\mathrm{r}} \int_{0}^{\mathrm{t}_{\mathrm{s}}^{\prime}} \mathrm{a}\left(\mathrm{e}^{(\mathrm{b}-\mathrm{r})\left(\mathrm{t}-\mathrm{t}_{\mathrm{s}}^{\prime}\right)}-\mathrm{e}^{\mathrm{bt}}\right) \mathrm{dt}\right.$ $=\left\{c_{0}+\frac{c_{2}+\beta c_{p}}{\beta+r}\left[\frac{1}{b+\beta}\left(e^{(b+\beta) t_{r}}-1\right)-\frac{1}{r-b}\left(1-e^{(b-r) t_{r}}\right)\right] a\right.$ $+\frac{c h_{2}+\beta c_{p}}{\beta+r} \int_{t_{s}^{\prime}}^{t_{r}^{\prime}} a\left(e^{(b+\beta)\left(t-t_{s}^{\prime}\right)}-e^{(b-r)\left(t-t_{s}^{\prime}\right)}\right) d t$

$+\frac{c h_{1}+\alpha c_{p}}{\alpha+r}\left[W-\frac{a}{r-b} e^{-r t_{r}}\left(e^{b t_{r}}-e^{b t_{0}-r \Delta_{1}}\right)\right]$ $\left.+\frac{\mathrm{ch}_{1}+\alpha \mathrm{c}_{\mathrm{p}}}{\alpha+\mathrm{r}} \int_{\mathrm{t}_{\mathrm{r}}^{\prime}}^{\mathrm{t}_{0}^{\prime}} \mathrm{a}\left(\mathrm{e}^{(\mathrm{b}+\alpha)\left(\mathrm{t}-\mathrm{t}_{\mathrm{s}}^{\prime}\right)}-\mathrm{e}^{(\mathrm{b}-\mathrm{r})\left(\mathrm{t}-\mathrm{t}_{\mathrm{s}}^{\prime}\right)}\right) \mathrm{dt}\right] /\left(\mathrm{t}_{\mathrm{s}}^{\prime}+\Delta_{3}+\Delta_{4}\right)$ 
Published Online July 2019 in IJEAST (http://www.ijeast.com)

$$
=e^{-r t_{s}^{\prime}}\left\{c_{0}+\frac{a c_{s}}{r}\left(\frac{e^{(b-r)\left(t-t_{s}^{\prime}\right)}}{b-r}-\frac{e^{b t}}{b}\right)_{0}^{t_{s}^{\prime}}\right.
$$

$+a \frac{\mathrm{ch}_{2}+\beta c_{p}}{\beta+r}\left(\frac{e^{(b+\beta)\left(t-t_{s}^{\prime}\right)}}{b+\beta}-\frac{e^{(b-r)\left(t-t_{s}^{\prime}\right)}}{b-r}\right)_{t_{s}^{\prime}}^{t_{r}^{\prime}}$

$\left.+a \frac{\operatorname{ch}_{1}+\alpha c_{p}}{\alpha+r}\left(\frac{e^{(b+\alpha)\left(t-t_{s}^{\prime}\right)}}{(b+\alpha)}-\frac{e^{(b-r)\left(t-t_{s}^{\prime}\right)}}{(b-r)}\right)_{t_{r}^{\prime}}^{t_{0}^{\prime}}\right] /\left(t_{s}^{\prime}+\Delta_{3}+\Delta_{4}\right)$

$=e^{-r t_{s}^{\prime}}\left\{c_{0}+\frac{a c_{s}}{r}\left(\frac{1}{b-r}-\frac{e^{b t_{s}^{\prime}}}{b}-\frac{e^{-(b-r) t_{s}^{\prime}}}{b-r}+\frac{1}{b}\right)\right.$

$+a \frac{c_{2}+\beta c_{p}}{\beta+r}\left(\frac{e^{(b+\beta)\left(t_{r}^{\prime}-t_{s}^{\prime}\right)}}{b+\beta}-\frac{e^{(b-r)\left(t_{r}^{\prime}-t_{s}^{\prime}\right)}}{b-r}-\frac{1}{b+\beta}+\frac{1}{b-r}\right)$

$+a \frac{\mathrm{ch}_{1}+\alpha c_{p}}{\alpha+r}\left(\frac{e^{(b+\alpha)\left(t_{0}^{\prime}-t_{s}^{\prime}\right)}}{b+\alpha}-\frac{e^{(b-r)\left(t_{0}^{\prime}-t_{s}^{\prime}\right)}}{b-r}-\frac{e^{(b+\alpha)\left(t_{r}^{\prime}-t_{s}^{\prime}\right)}}{b+\alpha}\right.$

$\left.\left.+\frac{\mathrm{e}^{(\mathrm{b}-\mathrm{r})\left(\mathrm{t}_{\mathrm{r}}^{\prime}-\mathrm{t}_{\mathrm{s}}^{\prime}\right)}}{\mathrm{b}-\mathrm{r}}\right)\right] /\left(\mathrm{t}_{\mathrm{s}}^{\prime}+\Delta_{3}+\Delta_{4}\right)$

$=e^{-r t_{s}^{\prime}}\left\{c_{0}+\frac{c_{s}}{r}\left(\frac{1}{r-b}\left(e^{(r-b) t_{s}^{\prime}}-1\right)-\frac{1}{b}\left(e^{b t_{s}^{\prime}}-1\right)\right) a\right.$

$+\frac{\mathrm{ch}_{2}+\beta \mathrm{c}_{\mathrm{p}}}{\beta+\mathrm{r}}\left(\frac{1}{\mathrm{~b}+\beta}\left(\mathrm{e}^{(\mathrm{b}+\beta) \Delta_{3}}-1\right)-\frac{1}{\mathrm{r}-\mathrm{b}}\left(1-\mathrm{e}^{-(\mathrm{r}-\mathrm{b}) \Delta_{3}}\right)\right) \mathrm{a}$

$+\frac{c h_{1}+\alpha c_{p}}{\alpha+r}\left(\frac{a}{(b+\alpha)}\left(e^{(b+\alpha)\left(t_{0}^{\prime}-t_{s}^{\prime}\right)}-e^{(b+\alpha)\left(t_{r}^{\prime}-t_{s}^{\prime}\right)}\right)\right.$

$$
\left.\left.-\frac{a}{r-b} e^{(b-r)}\left(e^{\left(\mathrm{t}_{\mathrm{r}}^{\prime}-\mathrm{t}_{\mathrm{s}}^{\prime}\right)}-\mathrm{e}^{\left(\mathrm{t}_{0}^{\prime}-\mathrm{t}_{\mathrm{s}}^{\prime}\right)}\right)\right)\right] /\left(\mathrm{t}_{\mathrm{s}}^{\prime}+\Delta_{3}+\Delta_{4}\right)
$$$$
=\mathrm{e}^{-\mathrm{rt}_{\mathrm{s}}^{\prime}}\left\{\mathrm{c}_{0}+\frac{\mathrm{c}_{\mathrm{s}}}{\mathrm{r}}\left(\frac{1}{\mathrm{r}-\mathrm{b}}\left(\mathrm{e}^{(\mathrm{r}-\mathrm{b}) \mathrm{t}_{\mathrm{s}}^{\prime}}-1\right)-\frac{1}{\mathrm{~b}}\left(\mathrm{e}^{\mathrm{bt_{ \textrm {s } } ^ { \prime }}}-1\right)\right) \mathrm{a}\right.
$$$$
+\frac{\mathrm{ch}_{2}+\beta \mathrm{c}_{\mathrm{p}}}{\beta+\mathrm{r}}\left(\frac{1}{\mathrm{~b}+\beta}\left(\mathrm{e}^{(\mathrm{b}+\beta) \Delta_{3}}-1\right)-\frac{1}{\mathrm{r}-\mathrm{b}}\left(1-\mathrm{e}^{-(\mathrm{r}-\mathrm{b}) \Delta_{3}}\right)\right) \mathrm{a}
$$

$$
+\frac{\mathrm{ch}_{1}+\alpha \mathrm{c}_{\mathrm{p}}}{\alpha+\mathrm{r}}
$$$$
\left.\left(\mathrm{W}-\frac{\mathrm{a}}{\mathrm{r}-\mathrm{b}} \mathrm{e}^{(\mathrm{b}-\mathrm{r}) \Delta_{3}}\left(1-\mathrm{e}^{(\mathrm{b}-\mathrm{r}) \Delta_{4}}\right)\right)\right] /\left(\mathrm{t}_{\mathrm{s}}^{\prime}+\Delta_{3}+\Delta_{4}\right)
$$

where $\mathrm{W}=\int_{\mathrm{t}_{\mathrm{r}}^{\prime}}^{\mathrm{t}_{0}^{\prime}} \mathrm{ae} \mathrm{e}^{(\mathrm{b}+\alpha) \mathrm{t}-\alpha \mathrm{t}_{\mathrm{s}}^{\prime}} \mathrm{dt}, \Delta_{3}=\mathrm{t}_{\mathrm{r}}^{\prime}-\mathrm{t}_{\mathrm{s}}^{\prime}$

and

$$
\Delta_{4}=\mathrm{t}_{0}^{\prime}-\mathrm{t}_{\mathrm{r}}^{\prime}=\operatorname{In}\left[1+\frac{(\mathrm{b}+\alpha)}{\mathrm{a}} \mathrm{We}^{-(\mathrm{b}+\alpha) \Delta_{3}}\right] /(\mathrm{b}+\alpha)
$$

The objective here is to determine the optimal values of $\mathrm{t}_{\mathrm{s}}^{\prime}$ and $\Delta_{4}$ in order to minimize the total relevant cost per unit time.

\section{SOLUTIONS OF THE MODEL}

Now, the optimal solutions for both model I and II will be established. Model I, from (19)

$$
\begin{aligned}
& {\left[\mathrm{e}^{-(\mathrm{r}-\mathrm{b}) \mathrm{t}_{\mathrm{r}}}\left(1-\mathrm{e}^{(\mathrm{b}-\mathrm{r}) \Delta_{1}}\right)-\frac{1}{\mathrm{r}-\mathrm{b}} \mathrm{e}^{-(\mathrm{r}-\mathrm{b}) \mathrm{t}_{\mathrm{r}}}\left(-\mathrm{e}^{(\mathrm{b}-\mathrm{r}) \Delta_{1}}\right)(\mathrm{b}-\mathrm{r}) \frac{\mathrm{d} \Delta_{1}}{\mathrm{dt}_{\mathrm{r}}}\right] \mathrm{a}} \\
& \Delta_{1}=\mathrm{t}_{\mathrm{o}}-\mathrm{t}_{\mathrm{r}}=\operatorname{In}\left[1+\frac{(\mathrm{b}+\alpha) \mathrm{W}}{\mathrm{a}} \mathrm{e}^{-(\mathrm{b}+\alpha) \mathrm{t}_{\mathrm{r}}}\right] /(\mathrm{b}+\boldsymbol{\alpha})
\end{aligned}
$$

$\frac{\mathrm{d} \Delta_{1}}{\mathrm{dt}_{\mathrm{r}}}=\frac{1}{(\alpha+\mathrm{b})}\left[\frac{-(\mathrm{b}+\alpha)^{2} \mathrm{e}^{-(\mathrm{b}+\alpha) \mathrm{t}_{\mathrm{r}}} \mathrm{W}}{1+\frac{(b+\alpha) \mathrm{W}}{\mathrm{a}} \mathrm{e}^{-(\mathrm{b}+\alpha) \mathrm{t}_{\mathrm{r}}}}\right] \frac{1}{\mathrm{a}}$ 
Published Online July 2019 in IJEAST (http://www.ijeast.com)

$$
\begin{aligned}
&= \frac{-(b+\alpha) W e^{-(b+\alpha) t_{r}}}{a\left[\frac{a+W(b+\alpha) e^{-(b+\alpha) t_{r}}}{a}\right]} \\
& \frac{d \Delta_{1}}{d t_{r}}=\frac{-(b+\alpha) W e^{-(b+\alpha) t_{r}}}{a+W(b+\alpha) e^{-(b+\alpha) t_{r}}}
\end{aligned}
$$

Also we have

$$
\begin{aligned}
& \Delta_{1}=\frac{1}{(b+\alpha)} \operatorname{In}\left[1+\frac{(b+\alpha) \mathrm{W}}{\mathrm{a}} \mathrm{e}^{-(\mathrm{b}+\alpha) \mathrm{t}_{\mathrm{r}}}\right] \\
& (\mathrm{b}+\alpha) \Delta_{1}=\operatorname{In}\left[\frac{\mathrm{a}+(\mathrm{b}+\alpha) \mathrm{We} \mathrm{e}^{-(\mathrm{b}+\alpha) \mathrm{t}_{\mathrm{r}}}}{\mathrm{a}}\right] \\
& \mathrm{e}^{(\mathrm{b}+\alpha) \Delta_{1}}=\frac{\mathrm{a}+(\mathrm{b}+\alpha) \mathrm{We} \mathrm{e}^{-(\mathrm{b}+\alpha) \mathrm{t}_{\mathrm{r}}}}{\mathrm{a}} \\
& \mathrm{e}^{-(\mathrm{b}+\alpha) \Delta_{1}}-1=\frac{\mathrm{a}}{\mathrm{a}+(\mathrm{b}+\alpha) \mathrm{We} \mathrm{e}^{-(\mathrm{b}+\alpha) \mathrm{t}_{\mathrm{r}}}-1} \\
& \therefore \quad \frac{\mathrm{d} \Delta_{1}}{\mathrm{dt}} \\
& =\frac{-(\mathrm{b}+\alpha) \mathrm{We} \mathrm{e}^{-(\mathrm{b}+\alpha) \Delta_{1}}-1=\frac{-(\mathrm{b}+\alpha) \mathrm{We}^{-(\mathrm{b}+\alpha) \mathrm{t}_{\mathrm{r}}}}{\mathrm{a}+(\mathrm{b}+\alpha) \mathrm{We}^{-(\mathrm{b}+\alpha) \mathrm{t}_{\mathrm{r}}}}}{\mathrm{a}+\mathrm{W}(\mathrm{b}+\alpha) \mathrm{e}^{-(\mathrm{b}+\alpha) \mathrm{t}_{\mathrm{r}}}=\mathrm{e}^{-(\mathrm{b}+\alpha) \Delta_{1}}-1}
\end{aligned}
$$

The necessary conditions for $\mathrm{Tc}_{1}$ in (18) to be minimum are

$$
\begin{gathered}
\frac{\partial \mathrm{Tc}_{1}}{\partial \mathrm{t}_{\mathrm{r}}}=\left\{\left[\frac{\mathrm{ch}_{2}+\beta \mathrm{c}_{\mathrm{p}}}{\beta+\mathrm{r}}\left(\mathrm{e}^{(\mathrm{b}+\beta) \mathrm{t}_{\mathrm{r}}}-\mathrm{e}^{(\mathrm{b}-\mathrm{r}) \mathrm{t}_{\mathrm{r}}}\right)\right] \mathrm{a}\right. \\
+\frac{\mathrm{ch}_{1}+\alpha \mathrm{c}_{\mathrm{p}}}{\alpha+\mathrm{r}}
\end{gathered}
$$$$
\left.+\frac{\mathrm{c}_{\mathrm{s}}}{\mathrm{r}} \mathrm{e}^{-\mathrm{r}\left(\mathrm{t}_{\mathrm{r}}+\Delta_{1}+\Delta_{2}\right)}(-\mathrm{r})\left(1+\frac{\mathrm{d} \Delta_{1}}{\mathrm{dt}}\right)\left[\frac{1}{\mathrm{r}}\right)\left(\mathrm{e}^{\mathrm{bt}+\mathrm{r} \Delta_{2}}-\mathrm{e}^{\mathrm{bt}}\right)-\Delta_{2}\right] \mathrm{a}
$$$$
\left.-\operatorname{Tc}_{1}\left(1+\frac{\mathrm{d} \Delta_{1}}{\mathrm{dt}_{\mathrm{r}}}\right)\right\} /\left(\mathrm{t}_{\mathrm{r}}+\Delta_{1}+\Delta_{2}\right)=0
$$

$$
\begin{aligned}
\frac{\partial \mathrm{Tc}_{1}}{\partial \mathrm{t}_{\mathrm{r}}}= & \left\{\left[\frac{\mathrm{ch}_{2}+\beta \mathrm{c}_{\mathrm{p}}}{\beta+\mathrm{r}}\left(\mathrm{e}^{(\mathrm{b}+\beta) \mathrm{t}_{\mathrm{r}}}-\mathrm{e}^{(\mathrm{b}-\mathrm{r}) \mathrm{t}_{\mathrm{r}}}\right)\right.\right. \\
& \left.+\frac{\mathrm{ch}_{1}+\alpha \mathrm{c}_{\mathrm{p}}}{\alpha+\mathrm{r}} \mathrm{e}^{-(\mathrm{r}-\mathrm{b}) \mathrm{t}_{\mathrm{r}}}\left(1-\mathrm{e}^{-(\alpha+\mathrm{r}) \Delta_{1}}\right)\right] \mathrm{a} \\
-\mathrm{c}_{\mathrm{s}} \mathrm{ae}^{-\mathrm{r}\left(\mathrm{t}_{\mathrm{r}}+\Delta_{1}+\Delta_{2}\right)}\left[\frac{1}{\mathrm{r}-\mathrm{b}}\left(\mathrm{e}^{\left.\mathrm{bt_{0 } + \mathrm { r } \Delta _ { 2 }}-\mathrm{e}^{\mathrm{b} \mathrm{t}_{\mathrm{s}}}\right)-\Delta_{2}}\right]\left(1+\frac{\mathrm{d} \Delta_{1}}{\mathrm{dt}_{\mathrm{r}}}\right)\right. & \\
& \left.-\mathrm{Tc}_{1}\left(1+\frac{\mathrm{d} \Delta_{1}}{\mathrm{dt}_{\mathrm{r}}}\right)\right\} /\left(\mathrm{t}_{\mathrm{r}}+\Delta_{1}+\Delta_{2}\right)=0
\end{aligned}
$$

and

$$
\begin{aligned}
& \frac{\partial \mathrm{Tc}_{1}}{\partial \Delta_{2}}=\left\{\frac{\mathrm{ac}_{\mathrm{s}}}{\mathrm{r}} \mathrm{e}^{-\mathrm{r}\left(\mathrm{t}_{\mathrm{r}}+\Delta_{1}+\Delta_{2}\right)}(-\mathrm{r})\left[\frac{1}{\mathrm{r}-\mathrm{b}}\left(\mathrm{e}^{\mathrm{bt}+\mathrm{r} \Delta_{2}}-\mathrm{e}^{\mathrm{bt}}\right)-\Delta_{2}\right]\right. \\
& \left.+\frac{\mathrm{c}_{\mathrm{s}}}{\mathrm{r}} \mathrm{e}^{-\mathrm{r}\left(\mathrm{t}_{\mathrm{r}}+\Delta_{1}+\Delta_{2}\right)}\left[\frac{\mathrm{r}}{\mathrm{r}-\mathrm{b}}\left(\mathrm{e}^{\mathrm{bt_{0 } + \mathrm { r } _ { 2 }}}-1\right)\right]-\mathrm{Tc}_{1}\right\} /\left(\mathrm{t}_{\mathrm{r}}+\Delta_{1}+\Delta_{2}\right)=0 \\
& \frac{\partial T \mathrm{c}_{1}}{\partial \Delta_{2}}=\left\{\mathrm { ac } _ { \mathrm { s } } \mathrm { e } ^ { - \mathrm { r } ( \mathrm { t } _ { \mathrm { r } } + \Delta _ { 1 } + \Delta _ { 2 } ) } \left[-\frac{1}{\mathrm{r}-\mathrm{b}} \mathrm{e}^{\mathrm{bt}_{0}+\mathrm{r} \Delta_{2}}\right.\right. \\
& \left.\left.+\frac{1}{\mathrm{r}-\mathrm{b}} \mathrm{e}^{\mathrm{bt_{ \textrm {s } }}}+\Delta_{2}+\frac{1}{\mathrm{r}-\mathrm{b}} \mathrm{e}^{\mathrm{bt}_{\mathrm{t}}+\mathrm{r} \Delta_{2}}-\frac{1}{\mathrm{r}}\right]-\mathrm{Tc}_{1}\right\} /\left(\mathrm{t}_{\mathrm{r}}+\Delta_{1}+\Delta_{2}\right)=0
\end{aligned}
$$$$
\frac{\partial \mathrm{Tc}_{1}}{\partial \Delta_{2}}=
$$$$
\left\{\mathrm{ac}_{\mathrm{s}} \mathrm{e}^{-\mathrm{r}\left(\mathrm{r}_{\mathrm{r}}+\Delta_{1}+\Delta_{2}\right)}\left[\frac{1}{\mathrm{r}-\mathrm{b}} \mathrm{e}^{\mathrm{b} \mathrm{s}_{\mathrm{s}}}+\Delta_{2}-\frac{1}{\mathrm{r}}\right]-\mathrm{Tc}_{1}\right\} /\left(\mathrm{t}_{\mathrm{r}}+\Delta_{1}+\Delta_{2}\right)=0
$$

From equation (23) and (24), the following can be easily obtained From (23)

$$
\begin{gathered}
\operatorname{Tc}_{1}\left(1+\frac{\mathrm{d} \Delta_{1}}{\mathrm{dt}_{\mathrm{r}}}\right)=\left[\frac{\mathrm{ch}_{2}+\beta \mathrm{c}_{\mathrm{p}}}{\beta+\mathrm{r}}\left(\mathrm{e}^{(\mathrm{b}+\beta) \mathrm{t}_{\mathrm{r}}}-\mathrm{e}^{(\mathrm{b}-\mathrm{r}) \mathrm{t}_{\mathrm{r}}}\right)\right. \\
\left.+\frac{\mathrm{ch}_{1}+\alpha \mathrm{c}_{\mathrm{p}}}{\alpha+\mathrm{r}} \mathrm{e}^{-(\mathrm{b}-\mathrm{r}) \mathrm{t}_{\mathrm{r}}}\left(1-\mathrm{e}^{-(\alpha+\mathrm{r}) \Delta_{1}}\right)\right] \mathrm{a} \\
-\mathrm{c}_{\mathrm{s}} \mathrm{ae}^{-\mathrm{r}\left(\mathrm{t}_{\mathrm{r}}+\Delta_{1}+\Delta_{2}\right)}
\end{gathered}
$$




$$
\begin{gathered}
{\left[\frac{1}{\mathrm{r}-\mathrm{b}}\left(\mathrm{e}^{\left(\mathrm{bt}_{0}+\mathrm{r} \Delta_{2}\right)}-\mathrm{e}^{\mathrm{bt}}\right)-\Delta_{2}\right]} \\
\left(1+\frac{\mathrm{d} \Delta_{1}}{\mathrm{dt}_{\mathrm{r}}}\right)
\end{gathered}
$$

$\mathrm{Tc}_{1} \mathrm{e}^{-(\mathrm{b}+\alpha) \Delta_{1}}=\left[\frac{\mathrm{ch}_{2}+\beta \mathrm{c}_{\mathrm{p}}}{\beta+\mathrm{r}}\left(\mathrm{e}^{(\mathrm{b}+\beta) \mathrm{t}_{\mathrm{r}}}-\mathrm{e}^{(\mathrm{b}-\mathrm{r}) \mathrm{t}_{\mathrm{r}}}\right)\right.$

$$
\left.+\frac{\mathrm{ch}_{1} \alpha \mathrm{c}_{\mathrm{p}}}{\alpha+\mathrm{r}} \mathrm{e}^{(\mathrm{b}-\mathrm{r}) \mathrm{t}_{\mathrm{r}}}\left(1-\mathrm{e}^{-(\alpha+\mathrm{r}) \Delta_{1}}\right)\right] \mathrm{a}
$$

$-c_{\mathrm{s}} a e^{-r\left(t_{r}+\Delta_{1}+\Delta_{2}\right)}$

$$
\left[\frac{1}{\mathrm{r}-\mathrm{b}}\left(\mathrm{e}^{\mathrm{bt} \mathrm{t}_{0}+\mathrm{r} \Delta_{2}}-\mathrm{e}^{\mathrm{bt}}\right)-\Delta_{2}\right] \mathrm{e}^{-(\mathrm{b}+\alpha) \Delta_{1}}
$$

and from (24)

$\mathrm{Tc}_{1}=\mathrm{c}_{\mathrm{s}} \mathrm{ae}^{-\mathrm{r}\left(\mathrm{t}_{\mathrm{r}}+\Delta_{1}+\Delta_{2}\right)}\left[\frac{1}{\mathrm{r}-\mathrm{b}} \mathrm{e}^{\mathrm{bt}_{\mathrm{s}}}+\Delta_{2}-\frac{1}{\mathrm{r}}\right]$

$\mathrm{Tc}_{1} \mathrm{e}^{-(\mathrm{b}+\alpha) \Delta_{1}}=\mathrm{ac}_{\mathrm{s}} \mathrm{e}^{-\mathrm{r}\left(\mathrm{t}_{\mathrm{r}}+\Delta_{1}+\Delta_{2}\right)}\left[\frac{1}{\mathrm{r}-\mathrm{b}} \mathrm{e}^{\mathrm{bt}}+\Delta_{2}-\frac{1}{\mathrm{r}}\right] \mathrm{e}^{-(\mathrm{b}+\alpha) \Delta_{1}}$ $\therefore$

$\mathrm{ac}_{\mathrm{s}} \mathrm{e}^{-\mathrm{r}\left(\mathrm{t}_{\mathrm{r}}+\Delta_{1}+\Delta_{2}\right)}\left[\frac{1}{\mathrm{r}-\mathrm{b}} \mathrm{e}^{\mathrm{bt}}+\Delta_{2}-\frac{1}{\mathrm{r}}\right] \mathrm{e}^{-(\mathrm{b}+\alpha) \Delta_{1}}$

$=\left[\frac{\mathrm{ch}_{2}+\beta c_{p}}{\beta+r}\left(e^{(b+\beta) t_{r}}-e^{(b-r) t_{r}}\right)+\frac{c h_{1}+\alpha c_{p}}{\alpha+r} e^{(b-r) t_{r}}\right.$

$$
\left.\left(1-\mathrm{e}^{-(\alpha+\mathrm{r}) \Delta_{1}}\right)\right] \mathrm{a}
$$

$-\mathrm{c}_{\mathrm{s}} \mathrm{ae}^{-\mathrm{r}\left(\mathrm{t}_{\mathrm{r}}+\Delta_{1}+\Delta_{2}\right)}$

$$
\left[\frac{1}{r-b}\left(e^{b t_{0}+r \Delta_{2}}-e^{b t_{s}}\right)-\Delta_{2}\right] e^{-(b+\alpha) \Delta_{1}}
$$

$c_{s} e^{-r\left(t_{r}+\Delta_{1}+\Delta_{2}\right)}\left[\frac{1}{r-b} e^{b t_{s}}+\Delta_{2}-\frac{1}{r}+\frac{1}{r-b}\left(e^{b t_{0}+r \Delta_{2}}\right)-\frac{e^{b t_{s}}}{r-b}-\Delta_{2}\right] e^{-(b+\alpha) \Delta_{1}}$

$$
\begin{aligned}
& =\left[\frac{c h_{2}+\beta c_{p}}{\beta+r}\left(e^{(b+\beta) t_{r}}-e^{(b-r) t_{r}}\right)+\frac{c h_{1}+\alpha c_{p}}{\alpha+r} e^{(b-r) t_{r}}\left(1-e^{-(\alpha+r) \Delta_{l}}\right)\right] \\
& c_{s} e^{-r\left(t_{r}+\Delta_{1}+\Delta_{2}\right)}\left[\frac{1}{r-b} e^{b t_{0}+r \Delta_{2}}-\frac{1}{r}\right] e^{-(b+\alpha) \Delta_{1}} \\
& =\left[\frac{c h_{2}+\beta c_{p}}{\beta+r}\left(e^{(b+\beta) t_{r}}-e^{(b-r) t_{r}}\right)+\frac{c h_{1}+\alpha c_{p}}{\alpha+r} e^{(b-r) t_{r}}\left(1-e^{-(\alpha+r) \Delta_{1}}\right)\right]
\end{aligned}
$$

This implies that

$$
\begin{aligned}
& c_{s} e^{-r\left(t_{r}+\Delta_{1}\right)}\left[\frac{e^{b t_{0}}}{r-b}-\frac{e^{-r \Delta_{2}}}{r}\right] e^{-(b+\alpha) \Delta_{1}} \\
& =e^{(b-r) t_{r}}\left[\frac{c h_{2}+\beta c_{p}}{\beta+r}\left(e^{(r+\beta) t_{r}}-1\right)+\frac{c h_{1}+\alpha c_{p}}{\alpha+r}\left(1-e^{-(\alpha+r) \Delta_{1}}\right)\right] \\
& c_{s}\left[\frac{e^{b t_{0}}}{r-b}-\frac{e^{-r \Delta_{2}}}{r}\right]=e^{r\left(t_{r}+\Delta_{1}\right)+(b+\alpha) \Delta_{1}+(b-r) t_{r}}
\end{aligned}
$$

$$
\begin{aligned}
& {\left[\frac{\mathrm{ch}_{2}+\beta \mathrm{c}_{\mathrm{p}}}{\beta+\mathrm{r}}\left(\mathrm{e}^{(\beta+\mathrm{r}) \mathrm{t}_{\mathrm{r}}}-1\right)+\frac{\mathrm{ch}_{1}+\alpha \mathrm{c}_{\mathrm{p}}}{\alpha+\mathrm{r}}\left(1-\mathrm{e}^{-(\alpha+\mathrm{r}) \Delta_{1}}\right)\right]} \\
& \mathrm{c}_{\mathrm{s}}\left[\frac{\mathrm{e}^{\mathrm{bt}}}{\mathrm{r}-\mathrm{b}}-\frac{\mathrm{e}^{-\mathrm{r} \Delta_{2}}}{\mathrm{r}}\right]=\mathrm{e}^{\mathrm{r} \Delta_{1}+\mathrm{b} \Delta_{1}+\alpha \Delta_{1}+\mathrm{bt} \mathrm{r}_{\mathrm{r}}}
\end{aligned}
$$

$\left[\frac{\mathrm{ch}_{2}+\beta \mathrm{c}_{\mathrm{p}}}{\beta+\mathrm{r}}\left(\mathrm{e}^{(\beta+\mathrm{r}) \mathrm{t}_{\mathrm{r}}}-1\right)+\frac{\mathrm{ch}_{1}+\alpha \mathrm{c}_{\mathrm{p}}}{\alpha+\mathrm{r}}\left(1-\mathrm{e}^{-(\alpha+\mathrm{r}) \Delta_{1}}\right)\right]$

$c_{s}\left[\frac{e^{b t_{0}}}{r-b}-\frac{e^{-r \Delta_{2}}}{r}\right]=e^{(r+b+\alpha) \Delta_{1}+b t_{r}}$

$\left[\frac{\mathrm{ch}_{2}+\beta \mathrm{c}_{\mathrm{p}}}{\beta+\mathrm{r}}\left(\mathrm{e}^{(\beta+\mathrm{r}) \mathrm{t}_{\mathrm{r}}}-1\right)+\frac{\mathrm{ch}_{1}+\alpha \mathrm{c}_{\mathrm{p}}}{\alpha+\mathrm{r}}\left(1-\mathrm{e}^{-(\alpha+\mathrm{r}) \Delta_{1}}\right)\right]$

Thus $\Delta_{2}$ is also a function of $t_{r}$ consequently, if $\mathrm{t}_{\mathrm{r}}$ is known, then $\Delta_{1}$ and $\Delta_{2}$ can be uniquely determined by (19) and (26), 
Published Online July 2019 in IJEAST (http://www.ijeast.com)

respectively. The solution that satisfies (23) and (24) minimizes $\mathrm{Tc}_{1}$. Similarly, for model II, from (21)

$$
\begin{gathered}
\Delta_{4}=\operatorname{In}\left[1+\frac{(b+\alpha)}{a} \mathrm{We}^{-(b+\alpha) \Delta_{3}}\right] /(b+\alpha) \\
\frac{\mathrm{d} \Delta_{4}}{\mathrm{~d} \Delta_{3}}=\frac{-(\mathrm{b}+\alpha)^{2} \mathrm{Wae}^{-(\mathrm{b}+\alpha) \Delta_{3}}}{(\mathrm{~b}+\alpha) \mathrm{a}\left[\mathrm{a}+(\mathrm{b}+\alpha) \mathrm{We} \mathrm{e}^{-(\mathrm{b}+\alpha) \Delta_{3}}\right]} \\
=\frac{-(\mathrm{b}+\alpha) \mathrm{We}^{-(\mathrm{b}+\alpha) \Delta_{3}}}{\mathrm{a}+(\mathrm{b}+\alpha) \mathrm{We}^{-(\mathrm{b}+\alpha) \Delta_{3}}}
\end{gathered}
$$

Also,

$$
\begin{array}{r}
\Delta_{4}=\operatorname{In}\left[1+\frac{(\mathrm{b}+\alpha) \mathrm{We}^{-(\mathrm{b}+\alpha) \Delta_{3}}}{\mathrm{a}}\right] /(\mathrm{b}+\alpha) \\
\mathrm{e}^{-(\mathrm{b}+\alpha) \Delta_{4}}-1=\frac{-(\mathrm{b}+\alpha) \mathrm{We}^{-(\mathrm{b}+\alpha) \Delta_{3}}}{\mathrm{a}+(\mathrm{b}+\alpha) \mathrm{We}^{-(\mathrm{b}+\alpha) \Delta_{3}}} \\
\frac{\mathrm{d} \Delta_{4}}{\mathrm{~d} \Delta_{3}} \\
=\frac{-(\mathrm{b}+\alpha) \mathrm{We}^{-(\mathrm{b}+\alpha) \Delta_{3}}}{\mathrm{a}+(\mathrm{b}+\alpha) \mathrm{We}^{-(\mathrm{b}+\alpha) \Delta_{3}}}=\mathrm{e}^{-(\mathrm{b}+\alpha) \Delta_{4}}-1
\end{array}
$$

The necessary conditions for $\mathrm{Tc}_{2}$ in (20) to be minimum are

$$
\begin{aligned}
& \frac{\partial \mathrm{Tc}_{2}}{\partial \mathrm{t}_{\mathrm{s}}^{\prime}}= \\
& \mathrm{e}^{-\mathrm{rt}_{\mathrm{s}}^{\prime}} \frac{\partial}{\partial \mathrm{t}_{\mathrm{s}}^{\prime}}\left\{\frac{\mathrm{c}_{\mathrm{s}}}{\mathrm{r}}\left[\frac{1}{\mathrm{r}-\mathrm{b}}\left(\mathrm{e}^{(\mathrm{r}-\mathrm{b}) \mathrm{t}_{\mathrm{s}}^{\prime}}-1\right)-\frac{1}{\mathrm{~b}}\left(\mathrm{e}^{\mathrm{bt_{ \textrm {s } } ^ { \prime }}}-1\right)\right] \mathrm{a}\right\} \\
& -\mathrm{re}^{-\mathrm{rt} \mathrm{r}_{\mathrm{s}}}\left\{\mathrm{c}_{0}+\frac{\mathrm{ch}_{2}+\beta \mathrm{c}_{\mathrm{p}}}{\beta+\mathrm{r}}\left[\frac{1}{\mathrm{~b}+\beta}\left(\mathrm{e}^{(\mathrm{b}+\beta) \Delta_{3}}-1\right)-\frac{1}{\mathrm{r}-\mathrm{b}}\left(1-\mathrm{e}^{-(\mathrm{r}-\mathrm{b}) \Delta_{3}}\right)\right] \mathrm{a}\right. \\
& +\frac{\mathrm{ch}_{1}+\alpha \mathrm{c}_{\mathrm{p}}}{\alpha+\mathrm{r}} \\
& \left.\left[\mathrm{W}-\frac{\mathrm{a}}{\mathrm{r}-\mathrm{b}} \mathrm{e}^{(\mathrm{b}-\mathrm{r}) \Delta_{3}}\left(1-\mathrm{e}^{(\mathrm{b}-\mathrm{r}) \Delta_{4}}\right)\right]\right\} /\left(\mathrm{t}_{\mathrm{s}}^{\prime}+\Delta_{3}+\Delta_{4}\right) \\
& -\mathrm{Tc}_{2} /\left(\mathrm{t}_{\mathrm{s}}^{\prime}+\Delta_{3}+\Delta_{4}\right)=0
\end{aligned}
$$

$$
\begin{gathered}
=\mathrm{e}^{-r \mathrm{rt}_{\mathrm{s}}^{\prime}}\left\{\mathrm{c}_{\mathrm{s}} \mathrm{t}_{\mathrm{s}}^{\prime} \mathrm{a}-\mathrm{r}\left[\mathrm{c}_{0}+\frac{\mathrm{ch}_{2}+\beta \mathrm{c}_{\mathrm{p}}}{\beta+\mathrm{r}}\left(\frac{1}{(\mathrm{~b}+\beta)}\left(\mathrm{e}^{(\mathrm{b}+\beta) \Delta_{3}}-1\right)\right.\right.\right. \\
\left.-\frac{1}{(\mathrm{r}-\mathrm{b})}\left(1-\mathrm{e}^{-(\mathrm{r}-\mathrm{b}) \Delta_{3}}\right)\right) \mathrm{a}+\frac{\mathrm{ch}_{1}+\alpha \mathrm{c}_{\mathrm{p}}}{\alpha+\mathrm{r}} \\
\left.\left.\left(\mathrm{w}-\frac{\mathrm{a}}{(\mathrm{r}-\mathrm{b})} \mathrm{e}^{(\mathrm{b}-\mathrm{r}) \Delta_{3}}\left(1-\mathrm{e}^{(\mathrm{b}-\mathrm{r}) \Delta_{4}}\right)\right)\right]\right\} /\left(\mathrm{t}_{\mathrm{s}}^{\prime}+\Delta_{3}+\Delta_{4}\right) \\
-\mathrm{Tc}_{2} /\left(\mathrm{t}_{\mathrm{s}}^{\prime}+\Delta_{3}+\Delta_{4}\right)=0
\end{gathered}
$$

and

$\frac{\partial T c_{2}}{\partial \Delta_{3}}=e^{-r t_{s}^{\prime}}\left\{\frac{\mathrm{ch}_{2}+\beta c_{p}}{\beta+r}\left[e^{(b+\beta) \Delta_{3}}-e^{-(r-b) \Delta_{3}}\right] a+\frac{\operatorname{ch}_{1}+\alpha c_{p}}{\alpha+r}\right.$

$\left.\left(\mathrm{ae}^{(\mathrm{b}-\mathrm{r}) \Delta_{3}}\left(1-\mathrm{e}^{(\mathrm{b}-\mathrm{r}) \Delta_{4}}\right)-\mathrm{ae}^{(\mathrm{b}-\mathrm{r}) \Delta_{3}} \mathrm{e}^{(\mathrm{b}-\mathrm{r}) \Delta_{4}} \frac{\mathrm{d} \Delta_{4}}{\mathrm{~d} \Delta_{3}}\right)\right\} /\left(\mathrm{t}_{\mathrm{s}}^{\prime}+\Delta_{3}+\Delta_{4}\right)$

$$
-\mathrm{Tc}_{2}\left(1+\frac{\mathrm{d} \Delta_{4}}{\mathrm{~d} \Delta_{3}}\right) /\left(\mathrm{t}_{\mathrm{s}}^{\prime}+\Delta_{3}+\Delta_{4}\right)=0
$$$$
=\mathrm{ee}^{-\mathrm{rt} \mathrm{r}_{\mathrm{s}}}\left[\frac{\mathrm{ch}_{2}+\beta \mathrm{c}_{\mathrm{p}}}{\beta+\mathrm{r}}\left(\mathrm{e}^{(\mathrm{b}+\beta) \Delta_{3}}-\mathrm{e}^{-(\mathrm{r}-\mathrm{b}) \Delta_{3}}\right)+\frac{\mathrm{ch}_{1}+\alpha \mathrm{c}_{\mathrm{p}}}{\alpha+\mathrm{r}} \mathrm{e}^{(\mathrm{b}-\mathrm{r}) \Delta_{3}}\right.
$$$$
\left.\left.\left(1-\mathrm{e}^{-(\alpha+\mathrm{r}) \Delta_{4}}\right)\right] /\left(\mathrm{t}_{\mathrm{s}}^{\prime}+\Delta_{3}+\Delta_{4}\right)\right]
$$$$
-\mathrm{Tc}_{2}\left(1+\frac{\mathrm{d} \Delta_{4}}{\mathrm{~d} \Delta_{3}}\right) /\left(\mathrm{t}_{\mathrm{s}}^{\prime}+\Delta_{3}+\Delta_{4}\right)=0
$$

Again from (28)

$$
\begin{aligned}
& \begin{aligned}
\mathrm{Tc}_{2}= & \mathrm{e}^{-\mathrm{rts}_{\mathrm{s}}^{\prime}}\left\{\mathrm{c}_{\mathrm{s}} \mathrm{t}_{\mathrm{s}}^{\prime} \mathrm{a}-\mathrm{r}\left[\mathrm{c}_{0}+\frac{\mathrm{ch}_{2}+\beta \mathrm{c}_{\mathrm{p}}}{\beta+\mathrm{r}}\left(\frac{1}{(\mathrm{~b}+\beta)}\left(\mathrm{e}^{(\mathrm{b}+\beta) \Delta_{3}}-1\right)\right.\right.\right. \\
& -\frac{1}{(\mathrm{r}-\mathrm{b})}\left(1-\mathrm{e}^{-(\mathrm{r}-\mathrm{b}) \Delta_{3}}\right) \mathrm{a}+\frac{\mathrm{ch}_{1}+\alpha \mathrm{c}_{\mathrm{p}}}{\alpha+\mathrm{r}} \\
& \left.\left.\left(\mathrm{W}-\frac{\mathrm{a}}{(\mathrm{r}-\mathrm{b})} \mathrm{e}^{(\mathrm{b}-\mathrm{r}) \Delta_{3}}\left(1-\mathrm{e}^{(\mathrm{b}-\mathrm{r}) \Delta_{4}}\right)\right)\right]\right\}
\end{aligned} \\
& \text { And from (29) }
\end{aligned}
$$


Published Online July 2019 in IJEAST (http://www.ijeast.com)

$$
\begin{array}{r}
\operatorname{Tc}_{2}\left(1+\frac{\mathrm{d} \Delta_{4}}{\mathrm{~d} \Delta_{3}}\right)=\mathrm{ae}^{-\mathrm{rt \prime} \mathrm{s}_{\mathrm{s}}}\left[\frac{\mathrm{ch}_{2}+\beta \mathrm{c}_{\mathrm{p}}}{\beta+\mathrm{r}}\left(\mathrm{e}^{(\mathrm{b}+\beta) \Delta_{3}}-\mathrm{e}^{-(\mathrm{r}-\mathrm{b}) \Delta_{3}}\right)\right. \\
\left.+\frac{\mathrm{ch}_{1}+\alpha \mathrm{c}_{\mathrm{p}}}{\alpha+\mathrm{r}} \mathrm{e}^{(\mathrm{b}-\mathrm{r}) \Delta_{3}}\left(1-\mathrm{e}^{-(\alpha+\mathrm{r}) \Delta_{4}}\right)\right]
\end{array}
$$

$\mathrm{Tc}_{2}=\mathrm{e}^{-\mathrm{rt} \mathrm{r}_{\mathrm{s}}}\left(\mathrm{a}+(\mathrm{b}+\alpha) \mathrm{We}^{-(\mathrm{b}+\alpha) \Delta_{3}}\right)\left[\frac{\mathrm{ch}_{2}+\beta \mathrm{c}_{\mathrm{p}}}{\beta+\mathrm{r}}\left(\mathrm{e}^{(\mathrm{b}+\beta) \Delta_{3}}-\mathrm{e}^{-(\mathrm{r}-\mathrm{b}) \Delta_{3}}\right)\right.$

$$
\frac{\mathrm{ch}_{1}+\alpha \mathrm{c}_{\mathrm{p}}}{\alpha+\mathrm{r}} \mathrm{e}^{(\mathrm{b}-\mathrm{r}) \Delta_{3}}\left(1-\mathrm{e}^{-(\alpha+\mathrm{r}) \Delta_{4}}\right)
$$

From (28) and (29)

$$
\begin{gathered}
\mathrm{c}_{\mathrm{s}} \mathrm{t}_{\mathrm{s}}^{\prime} \mathrm{a}=\mathrm{r}\left\{\mathrm{c}_{0}+\frac{\mathrm{ch}_{2}+\beta \mathrm{c}_{\mathrm{p}}}{\beta+\mathrm{r}}\left[\frac{1}{(\mathrm{~b}+\beta)}\left(\mathrm{e}^{(\mathrm{b}+\beta) \Delta_{3}}-1\right)\right.\right. \\
\left.-\frac{1}{(\mathrm{r}-\mathrm{b})}\left(1-\mathrm{e}^{\left.-(\mathrm{r}-\mathrm{b}) \Delta_{3}\right)}\right)\right] \mathrm{a} \\
+\frac{\mathrm{ch}_{1}+\alpha \mathrm{c}_{\mathrm{p}}}{\alpha+\mathrm{r}}
\end{gathered}
$$$$
\left.\left[\mathrm{W}-\frac{\mathrm{a}}{(\mathrm{r}-\mathrm{b})} \mathrm{e}^{(\mathrm{b}-\mathrm{r}) \Delta_{3}}\left(1-\mathrm{e}^{(\mathrm{b}-\mathrm{r}) \Delta_{4}}\right)\right]\right\}
$$$$
+\left[\left(a+(b+\alpha) W e^{-(b+\alpha) \Delta_{3}}\right)\right]\left[\frac{c_{2}+\beta c_{p}}{\beta+r}\left(e^{(b+\beta) \Delta_{3}}-e^{-(r-b) \Delta_{3}}\right)\right.
$$$$
\left.+\frac{\mathrm{ch}_{1}+\alpha c_{\mathrm{p}}}{\alpha+\mathrm{r}} \mathrm{e}^{(\mathrm{b}-\mathrm{r}) \Delta_{3}}\left(1-\mathrm{e}^{-(\alpha+\mathrm{r}) \Delta_{4}}\right)\right]
$$$$
\mathrm{c}_{\mathrm{s}} \mathrm{t}_{\mathrm{s}}^{\prime} \mathrm{a}=\mathrm{rc}_{0}+\frac{\mathrm{ch}_{2}+\beta \mathrm{c}_{\mathrm{p}}}{\beta+\mathrm{r}}\left[\frac{\mathrm{ar}}{\mathrm{b}+\beta}\left(\mathrm{e}^{(\mathrm{b}+\beta) \Delta_{3}}-1\right)-\frac{\mathrm{ar}}{\mathrm{r}-\mathrm{b}}\left(1-\mathrm{e}^{-(\mathrm{r}-\mathrm{b}) \Delta_{3}}\right)\right.
$$$$
\left.+\mathrm{ae}^{(\mathrm{b}+\beta) \Delta_{3}}-\mathrm{ae}^{(\mathrm{r}-\mathrm{b}) \Delta_{3}}+(\mathrm{b}+\alpha) \mathrm{W} \mathrm{e}^{-(\mathrm{b}+\alpha) \Delta_{3}}\left(\mathrm{e}^{(\mathrm{b}+\beta) \Delta_{3}}-\mathrm{e}^{-(\mathrm{r}-\mathrm{b}) \Delta_{3}}\right)\right]
$$

$$
+\frac{\mathrm{ch}_{1}+\alpha \mathrm{c}_{\mathrm{p}}}{\alpha+\mathrm{r}}
$$

$$
\begin{array}{r}
{\left[\mathrm{rW}-\frac{\mathrm{ar}}{\mathrm{r}-\mathrm{b}} \mathrm{e}^{(\mathrm{b}-\mathrm{r}) \Delta_{3}}\left(-\mathrm{e}^{(\mathrm{b}-\mathrm{r}) \Delta_{4}}\right)\right.} \\
\left.+\mathrm{e}^{(\mathrm{b}-\mathrm{r}) \Delta_{3}}\left(1-\mathrm{e}^{-(\alpha+\mathrm{r}) \Delta_{4}}\right)\right]
\end{array}
$$

Which implies that $\mathrm{t}_{\mathrm{s}}^{\prime}$ is a function of $\Delta_{3}$. Consequently, if $\Delta_{3}$ is known, then $\mathrm{t}_{\mathrm{s}}^{\prime}$ and $\Delta_{4}$ can be uniquely determined by (30) and (21), respectively. The solution that satisfies (28) and (29) minimizes $\mathrm{Tc}_{2}$.

Taking the second partial derivatives of $\mathrm{Tc}_{1}$ with respect to $\mathrm{t}_{\mathrm{r}}$ and $\Delta_{2}$ respectively, by (23) \& (25), we have

$$
\frac{\partial^{2} \mathrm{Tc}_{1}}{\partial \mathrm{t}_{\mathrm{r}}{ }^{2}}=\left\{\frac{\mathrm{ch}_{2}+\beta \mathrm{c}_{\mathrm{p}}}{\beta+\mathrm{r}}\left[(\mathrm{b}+\beta) \mathrm{e}^{(\mathrm{b}+\beta) \mathrm{t}_{\mathrm{r}}}-(\mathrm{b}-\mathrm{r}) \mathrm{e}^{(\mathrm{b}-\mathrm{r}) \mathrm{t}_{\mathrm{r}}}\right] \mathrm{a}\right.
$$$$
+\frac{\mathrm{ch}_{1}+\alpha \mathrm{c}_{\mathrm{p}}}{\alpha+\mathrm{r}}
$$

$\left[\mathrm{e}^{(\mathrm{b}-\mathrm{r}) \mathrm{t}_{\mathrm{r}}} \mathrm{e}^{-(\alpha+\mathrm{r}) \Delta_{1}}(\alpha+\mathrm{r}) \frac{\mathrm{d} \Delta_{1}}{\mathrm{dt}_{\mathrm{r}}}+(\mathrm{b}-\mathrm{r}) \mathrm{e}^{(\mathrm{b}-\mathrm{r}) \mathrm{t}_{\mathrm{r}}}\left(1-\mathrm{e}^{-(\alpha+\mathrm{r}) \Delta_{1}}\right)\right] \mathrm{a}$

$+c_{s} a e^{-r\left(t_{r}+\Delta_{1}+\Delta_{2}\right)}\left[\frac{1}{r-b}\left(e^{b t_{0}+r \Delta_{2}}-e^{b t_{s}}\right)-\Delta_{2}\right]$

$$
\begin{aligned}
& \mathrm{r}\left(1+\frac{\mathrm{d} \Delta_{1}}{\mathrm{dt}_{\mathrm{r}}}\right)^{2} \\
& -\mathrm{c}_{\mathrm{s}} \mathrm{ae}^{-\mathrm{r}\left(\mathrm{t}_{\mathrm{r}}+\Delta_{1}+\Delta_{2}\right)}
\end{aligned}
$$

$$
\begin{array}{r}
{\left[\frac{1}{\mathrm{r}-\mathrm{b}}\left(\mathrm{e}^{\mathrm{bt} \mathrm{t}_{0}+\mathrm{r} \Delta_{2}}-\mathrm{e}^{\mathrm{bt_{ \textrm {s } }}}\right)-\Delta_{2}\right] \frac{\mathrm{d}^{2} \Delta_{1}}{\mathrm{dt}_{\mathrm{r}}^{2}}} \\
-\mathrm{Tc}_{1} \frac{\mathrm{d}^{2} \Delta_{1}}{\mathrm{dt}_{\mathrm{r}}^{2}}-\left(1+\frac{\mathrm{d} \Delta_{1}}{\mathrm{dt}_{\mathrm{r}}}\right) \frac{\partial \mathrm{Tc} \mathrm{c}_{1}}{\partial \mathrm{t}_{\mathrm{r}}}
\end{array}
$$

$$
\begin{gathered}
\left.-\frac{\partial \mathrm{Tc}_{1}}{\partial \mathrm{t}_{\mathrm{r}}}\left(1+\frac{\mathrm{d} \Delta_{1}}{\mathrm{dt}}\right)\right\} /\left(\mathrm{t}_{\mathrm{r}}+\Delta_{1}+\Delta_{2}\right) \\
\frac{\partial^{2} \mathrm{Tc}_{1}}{\partial \mathrm{t}_{\mathrm{r}}^{2}}=\left\{\frac{\mathrm{ch}_{2}+\beta \mathrm{c}_{\mathrm{p}}}{\beta+\mathrm{r}}\left[(\mathrm{b}+\beta) \mathrm{e}^{(\mathrm{b}+\beta) \mathrm{t}_{\mathrm{r}}}-(\mathrm{b}-\mathrm{r}) \mathrm{e}^{(\mathrm{b}-\mathrm{r}) \mathrm{t}_{\mathrm{r}}}\right] \mathrm{a}\right. \\
+\mathrm{a} \frac{\mathrm{ch}_{1}+\alpha \mathrm{c}_{\mathrm{p}}}{\alpha+\mathrm{r}} \mathrm{e}^{(\mathrm{b}-\mathrm{r}) \mathrm{t}_{\mathrm{r}}}\left[(\mathrm{b}-\mathrm{r})\left(1-\mathrm{e}^{-(\alpha+\mathrm{r}) \Delta_{1}}\right)+(\alpha+\mathrm{r}) \mathrm{e}^{-(\alpha+\mathrm{r}) \Delta_{1}} \frac{\mathrm{d} \Delta_{1}}{\mathrm{dt}}\right] \mathrm{a}
\end{gathered}
$$


$+c_{\mathrm{s}} a e^{-r\left(t_{\mathrm{r}}+\Delta_{1}+\Delta_{2}\right)}\left[\frac{\mathrm{r}}{\mathrm{r}-\mathrm{b}}\left(\mathrm{e}^{\mathrm{bt} \mathrm{t}_{0}+\mathrm{r} \Delta_{2}}-\mathrm{e}^{\mathrm{bt}}\right)-\mathrm{r} \Delta_{2}\right]$

$$
\left(1+\frac{\mathrm{d} \Delta_{1}}{\mathrm{dt}_{\mathrm{r}}}\right)^{2}
$$

$$
+c_{s} a e^{-r\left(t_{r}+\Delta_{1}+\Delta_{2}\right)} \frac{1}{r-b}\left(e^{b t_{0}+r \Delta_{2}}-e^{b t_{s}}\right)
$$

$(\mathrm{b}+\alpha) \mathrm{e}^{-(\mathrm{b}+\alpha) \Delta_{1}} \frac{\mathrm{d} \Delta_{1}}{\mathrm{dt}_{\mathrm{r}}}$

$$
-c_{\mathrm{s}} \mathrm{a} \mathrm{e}^{-\mathrm{r}\left(\mathrm{t}_{\mathrm{r}}+\Delta_{1}+\Delta_{2}\right)}
$$

$\Delta_{2}(\mathrm{~b}+\alpha) \mathrm{e}^{-(\mathrm{b}+\alpha) \Delta_{1}} \frac{\mathrm{d} \Delta_{1}}{\mathrm{dt}_{\mathrm{r}}}$

$+\mathrm{c}_{\mathrm{s}} \mathrm{ae}^{-\mathrm{r}\left(\mathrm{t}_{\mathrm{r}}+\Delta_{1}+\Delta_{2}\right)}\left(\frac{1}{\mathrm{r}-\mathrm{b}} \mathrm{e}^{\mathrm{bt}}+\Delta_{2}-\frac{1}{\mathrm{r}}\right)$

$\left.(\mathrm{b}+\alpha) \mathrm{e}^{-(\mathrm{b}+\alpha) \Delta_{1}} \frac{\mathrm{d} \Delta_{1}}{\mathrm{dt}_{\mathrm{r}}}\right\} /\left(\mathrm{t}_{\mathrm{r}}+\Delta_{1}+\Delta_{2}\right)$

$\frac{\partial^{2} \mathrm{Tc}_{1}}{\partial \mathrm{t}_{\mathrm{r}}^{2}}=\mathrm{a}\left\{\frac{\mathrm{ch}_{2}+\beta \mathrm{c}_{\mathrm{p}}}{\beta+\mathrm{r}}\left[(\mathrm{b}+\beta) \mathrm{e}^{(\mathrm{b}+\beta) \mathrm{t}_{\mathrm{r}}}-(\mathrm{b}-\mathrm{r}) \mathrm{e}^{(\mathrm{b}-\mathrm{r}) \mathrm{t}_{\mathrm{r}}}\right]\right.$

$+\frac{\mathrm{ch}_{1}+\alpha c_{\mathrm{p}}}{\alpha+\mathrm{r}} \mathrm{e}^{(\mathrm{b}-\mathrm{r}) \mathrm{t}_{\mathrm{r}}}\left[(\mathrm{b}-\mathrm{r})\left(1-\mathrm{e}^{-(\alpha+\mathrm{r}) \Delta_{1}}\right)+(\alpha+\mathrm{r}) \mathrm{e}^{-(\alpha+\mathrm{r}) \Delta_{1}} \frac{\mathrm{d} \Delta_{1}}{\mathrm{dt}_{\mathrm{r}}}\right.$

$+c_{s} e^{-r\left(t_{r}+\Delta_{1}+\Delta_{2}\right)}\left[\frac{r}{r-b}\left(e^{b t_{0}+r \Delta_{2}}-e^{b t_{s}}\right)-r \Delta_{2}\right]$

$\left(1+\frac{\mathrm{d} \Delta_{1}}{\mathrm{dt}_{\mathrm{r}}}\right)^{2}$

$+c_{s} e^{-r\left(t_{r}+\Delta_{1}+\Delta_{2}\right)}\left(e^{b t_{0}+r \Delta_{2}}-e^{b t_{s}}\right)$

$\left(\frac{\mathrm{b}+\alpha}{\mathrm{r}-\mathrm{b}}\right) \mathrm{e}^{-(\mathrm{b}+\alpha) \Delta_{1}} \frac{\mathrm{d} \Delta_{1}}{\mathrm{dt}_{\mathrm{r}}}$

$+c_{s} e^{-r\left(t_{r}+\Delta_{1}+\Delta_{2}\right)}(b+\alpha) e^{-(b+\alpha) \Delta_{1}}$

$$
\begin{array}{r}
\left.\frac{\mathrm{d} \Delta_{1}}{\mathrm{dt}_{\mathrm{r}}}\left(\frac{1}{\mathrm{r}-\mathrm{b}} \mathrm{e}^{\mathrm{bt}}-\frac{1}{\mathrm{r}}\right)\right\} /\left(\mathrm{t}_{\mathrm{r}}+\Delta_{1}+\Delta_{2}\right) \\
\frac{\partial^{2} \mathrm{Tc}_{1}}{\partial \mathrm{t}_{\mathrm{r}}^{2}}=\mathrm{a}\left\{\frac{\mathrm{ch}_{2}+\beta \mathrm{c}_{\mathrm{p}}}{\beta+\mathrm{r}}\left[(\mathrm{b}+\beta) \mathrm{e}^{(\mathrm{b}+\beta) \mathrm{t}_{\mathrm{r}}}-(\mathrm{b}-\mathrm{r}) \mathrm{e}^{(\mathrm{b}-\mathrm{r}) \mathrm{t}_{\mathrm{r}}}\right]\right.
\end{array}
$$$$
+\frac{\mathrm{ch}_{1}+\alpha \mathrm{c}_{\mathrm{p}}}{\alpha+\mathrm{r}} \mathrm{e}^{(\mathrm{b}-\mathrm{r}) \mathrm{t}_{\mathrm{r}}}\left[(\mathrm{b}-\mathrm{r})\left(1-\mathrm{e}^{-(\alpha+\mathrm{r}) \Delta_{1}}\right)+(\alpha+\mathrm{r}) \mathrm{e}^{-(\alpha+\mathrm{r}) \Delta_{1}} \frac{\mathrm{d} \Delta_{1}}{\mathrm{dt}_{\mathrm{r}}}\right]
$$$$
+c_{s} e^{-r\left(t_{r}+\Delta_{1}+\Delta_{2}\right)}\left[\frac{r}{r-b}\left(e^{b t_{0}+r \Delta_{2}}-e^{b t_{s}}\right)-r \Delta_{2}\right]
$$

$$
\left(1+\frac{\mathrm{d} \Delta_{1}}{d t_{\mathrm{r}}}\right)^{2}+\mathrm{c}_{\mathrm{s}} \mathrm{e}^{-\mathrm{r}\left(\mathrm{t}_{\mathrm{r}}+\Delta_{1}+\Delta_{2}\right)}\left(\mathrm{e}^{\mathrm{bt} \mathrm{t}_{0}+\mathrm{r} \Delta_{2}}-\mathrm{e}^{\mathrm{bt_{ \textrm {s } }}}\right)
$$

$\left(\frac{\mathrm{b}+\alpha}{\mathrm{r}-\mathrm{b}}\right)\left(1+\frac{\mathrm{d} \Delta_{1}}{\mathrm{dt}_{\mathrm{r}}}\right) \frac{\mathrm{d} \Delta_{1}}{\mathrm{dt}_{\mathrm{r}}}+\mathrm{c}_{\mathrm{s}} \mathrm{e}^{-\mathrm{r}\left(\mathrm{t}_{\mathrm{r}}+\Delta_{1}+\Delta_{2}\right)}(\mathrm{b}+\alpha) \mathrm{e}^{-(\mathrm{b}+\alpha) \Delta_{1}}$

$$
\begin{gathered}
\left.\frac{\mathrm{d} \Delta_{1}}{\mathrm{dt}_{\mathrm{r}}}\left(\frac{1}{\mathrm{r}-\mathrm{b}} \mathrm{e}^{\mathrm{bt}}-\frac{1}{\mathrm{r}}\right)\right\} /\left(\mathrm{t}_{\mathrm{r}}+\Delta_{1}+\Delta_{2}\right) \\
\frac{\partial^{2} \mathrm{Tc}_{1}}{\partial \mathrm{t}_{\mathrm{r}}^{2}}=\mathrm{a}\left\{\frac{\mathrm{ch}_{2}+\beta \mathrm{c}_{\mathrm{p}}}{\beta+\mathrm{r}}\left[(\mathrm{b}+\beta) \mathrm{e}^{(\mathrm{b}+\beta) \mathrm{t}_{\mathrm{r}}}-(\mathrm{b}-\mathrm{r}) \mathrm{e}^{(\mathrm{b}-\mathrm{r}) \mathrm{t}_{\mathrm{r}}}\right]\right.
\end{gathered}
$$

$+\frac{\mathrm{ch}_{1}+\alpha \mathrm{c}_{\mathrm{p}}}{\alpha+\mathrm{r}}(\mathrm{b}-\mathrm{r}) \mathrm{e}^{(\mathrm{b}-\mathrm{r}) \mathrm{t}_{\mathrm{r}}}\left(1-\mathrm{e}^{-(\alpha+\mathrm{r}) \Delta_{1}}\right)$

$$
+\left(\mathrm{ch}_{1}+\alpha c_{p}\right) e^{-(\alpha+r) \Delta_{1}}\left(e^{-(b+\alpha) \Delta_{1}}-1\right)
$$

$+c_{s} e^{-r\left(t_{r}+\Delta_{1}+\Delta_{2}\right)} \frac{r}{r-b}\left(e^{b t_{0}+r \Delta_{2}}-e^{b t_{s}}\right) e^{-2(b+\alpha) \Delta_{1}}$ 
Published Online July 2019 in IJEAST (http://www.ijeast.com)

$-c_{s} e^{-r\left(t_{r}+\Delta_{1}+\Delta_{2}\right)} r \Delta_{2} e^{-2(b+\alpha) \Delta_{1}}+c_{s} e^{-r\left(t_{r}+\Delta_{1}+\Delta_{2}\right)}\left(e^{b t_{0}+r \Delta_{2}}-e^{b t_{s}}\right)$

$$
\begin{aligned}
& \frac{\partial^{2} \mathrm{Tc}_{1}}{\partial \mathrm{t}_{\mathrm{r}}^{2}}=\mathrm{a}\left\{\left(\mathrm{ch}_{2}+\beta \mathrm{c}_{\mathrm{p}}\right) \mathrm{e}^{(\mathrm{b}+\beta) \mathrm{t}_{\mathrm{r}}}-\left(\mathrm{ch}_{1}+\alpha \mathrm{c}_{\mathrm{p}}\right) \mathrm{e}^{-(\alpha+\mathrm{r}) \Delta_{1}}\left(1-\mathrm{e}^{-(\mathrm{b}+\alpha) \Delta_{1}}\right)\right. \\
& +c_{s} e^{-r\left(t_{r}+\Delta_{1}+\Delta_{2}\right)} e^{-(b+\alpha) \Delta_{1}}\left[-e^{b t_{0}+r \Delta_{2}}-\frac{b-r}{r}+\frac{r}{r-b} e^{b t_{0}+r \Delta_{2}}\right. \\
& e^{-(b+\alpha) \Delta_{1}}-\frac{r}{r-b} e^{b t_{s}} e^{-(b+\alpha) \Delta_{1}} \\
& -\left(\frac{b+a}{r-b}\right) \\
& \left(1-e^{-(b+\alpha) \Delta_{1}}\right)\left(e^{b t_{0}+r \Delta_{2}}-e^{b t_{s}}\right)+(b+\alpha)\left(e^{-(b+\alpha) \Delta_{1}}-1\right)
\end{aligned}
$$$$
\left(\frac{\mathrm{b}+\alpha}{\mathrm{r}-\mathrm{b}}\right)\left(\mathrm{e}^{-(\mathrm{b}+\alpha) \Delta_{1}}-1\right) \mathrm{e}^{-(\mathrm{b}+\alpha) \Delta_{1}}-\mathrm{c}_{\mathrm{s}} \mathrm{e}^{-\mathrm{r}\left(\mathrm{t}_{\mathrm{r}}+\Delta_{1}+\Delta_{2}\right)}(\mathrm{b}+\alpha) \mathrm{e}^{-(\mathrm{b}+\alpha) \Delta_{1}}
$$$$
\left.\left(\mathrm{e}^{-(\mathrm{b}+\alpha) \Delta_{1}}-1\right)\left(\frac{1}{\mathrm{r}-\mathrm{b}} \mathrm{e}^{\mathrm{bt}}-\frac{1}{\mathrm{r}}\right)\right\} /\left(\mathrm{t}_{\mathrm{r}}+\Delta_{1}+\Delta_{2}\right)
$$$$
\frac{\partial^{2} \mathrm{Tc}_{1}}{\partial \mathrm{t}_{\mathrm{r}}^{2}}=\mathrm{a}\left\{\frac{\mathrm{ch} \mathrm{h}_{2}+\beta \mathrm{c}_{\mathrm{p}}}{\beta+\mathrm{r}}\left[(\mathrm{b}+\beta) \mathrm{e}^{(\mathrm{b}+\beta) \mathrm{t}_{\mathrm{r}}}-(\mathrm{b}-\mathrm{r}) \mathrm{e}^{(\mathrm{b}-\mathrm{r}) \mathrm{t}_{\mathrm{r}}}\right]\right.
$$$$
+c_{s} e^{-r\left(t_{r}+\Delta_{1}+\Delta_{2}\right)}(b-r)\left(\frac{1}{r-b} e^{b t_{0}+r \Delta_{2}}-\frac{1}{r}\right) e^{-(b+\alpha) \Delta_{1}}
$$

$$
\begin{aligned}
& -\frac{c h_{2}+\beta c_{p}}{\beta+r}(b-r)\left(e^{(b+\beta) t_{r}}-e^{(b-r) t_{r}}\right) \\
& +\left(c h_{1}+\alpha c_{p}\right) e^{-(\alpha+r) \Delta_{1}}\left(e^{-(b+\alpha) \Delta_{1}}-1\right)
\end{aligned}
$$$$
\left.\left.\left(\frac{1}{\mathrm{r}-\mathrm{b}} \mathrm{e}^{\mathrm{bt}}-\frac{1}{\mathrm{r}}\right)\right]-\mathrm{c}_{\mathrm{s}} \mathrm{e}^{-\mathrm{r}\left(\mathrm{t}_{\mathrm{r}}+\Delta_{1}+\Delta_{2}\right)} \mathrm{r} \Delta_{2} \mathrm{e}^{-2(\mathrm{~b}+\alpha) \Delta_{1}}\right\} /\left(\mathrm{t}_{\mathrm{r}}+\Delta_{1}+\Delta_{2}\right)
$$

$\frac{\partial^{2} \mathrm{Tc}_{1}}{\partial \mathrm{t}_{\mathrm{r}}^{2}}=\mathrm{a}\left\{\left(\mathrm{ch}_{2}+\beta \mathrm{c}_{\mathrm{p}}\right) \mathrm{e}^{(\mathrm{b}+\beta) \mathrm{t}_{\mathrm{r}}}-\left(\mathrm{ch}_{1}+\alpha \mathrm{c}_{\mathrm{p}}\right) \mathrm{e}^{-(\alpha+\mathrm{r}) \Delta_{1}}\left(1-\mathrm{e}^{-(\alpha+\mathrm{r}) \Delta_{1}}\right)\right.$

$+c_{s} e^{-r\left(t_{r}+\Delta_{1}+\Delta_{2}\right)} \frac{r}{r-b}\left(e^{b t_{0}+r \Delta_{2}}-e^{b t_{s}}\right) e^{-2(b+\alpha) \Delta_{1}}$

$$
\begin{aligned}
& +c_{s} e^{-r\left(t_{r}+\Delta_{1}+\Delta_{2}\right)} e^{-(b+\alpha) \Delta_{1}}\left[-e^{b t_{0}+r \Delta_{2}}-\frac{b-r}{r}+\frac{r}{r-b} e^{b t_{0}+r \Delta_{2}}\right. \\
& e^{-(b+\alpha) \Delta_{1}}-\frac{r}{r-b} e^{b t_{s}} e^{-(b+\alpha) \Delta_{1}} \\
& -\left(\frac{b+\alpha}{r-b}\right) e^{b t_{0}+r \Delta_{2}}
\end{aligned}
$$$$
-c_{s} e^{-r\left(t_{r}+\Delta_{1}+\Delta_{2}\right)}\left(e^{b t_{0}+r \Delta_{2}}-e^{b t_{s}}\right)
$$

$\left(\frac{b+a}{r-b}\right)$

$\left(1-\mathrm{e}^{-(\mathrm{b}+\alpha) \Delta_{1}}\right) \mathrm{e}^{-(\mathrm{b}+\alpha) \Delta_{1}}-\mathrm{c}_{\mathrm{s}} \mathrm{e}^{-\mathrm{r}\left(\mathrm{t}_{\mathrm{r}}+\Delta_{1}+\Delta_{2}\right)} \mathrm{r} \Delta_{2} \mathrm{e}^{-2(\mathrm{~b}+\alpha) \Delta_{1}}$

$$
+\frac{b+\alpha}{r-b} e^{b t_{s}}+\frac{b+a}{r-b} e^{-(b+\alpha) \Delta_{1}} e^{b t_{0}+r \Delta_{2}}
$$

$+c_{s} e^{-r\left(t_{r}+\Delta_{1}+\Delta_{2}\right)}(b+\alpha) e^{-(b+\alpha) \Delta_{1}}\left(e^{-(b+\alpha) \Delta_{1}}-1\right)$

$$
\begin{aligned}
& -\left(\frac{b+\alpha}{r-b}\right) e^{-(b+\alpha) \Delta_{1}} e^{b t_{s}}+\left(\frac{b+\alpha}{r-b}\right) e^{-(b+\alpha) \Delta_{1}} e^{b t_{s}} \\
& \left.-\frac{(b+\alpha) e^{-(b+\alpha) \Delta_{1}}}{r}-\frac{(b+\alpha)}{r-b} e^{b t_{s}}+\frac{b+\alpha}{r}\right]
\end{aligned}
$$$$
\left.\left(\frac{1}{r-b} e^{b t_{s}}-\frac{1}{r}\right)\right\} /\left(t_{r}+\Delta_{1}+\Delta_{2}\right)
$$ 
$\left.-\mathrm{c}_{\mathrm{s}} \mathrm{e}^{-\mathrm{r}\left(\mathrm{t}_{\mathrm{r}}+\Delta_{1}+\Delta_{2}\right)} \mathrm{r} \Delta_{2} \mathrm{e}^{-2(\mathrm{~b}+\alpha) \Delta_{1}}\right\} /\left(\mathrm{t}_{\mathrm{r}}+\Delta_{1}+\Delta_{2}\right)$

$=\mathrm{a}\left(\mathrm{ch}_{2}+\beta \mathrm{c}_{\mathrm{p}}\right) \mathrm{e}^{(\mathrm{b}+\beta) \mathrm{t}_{\mathrm{r}}}-\left(\mathrm{ch}_{1}+\alpha \mathrm{c}_{\mathrm{p}}\right) \mathrm{e}^{-(\alpha+\mathrm{r}) \Delta_{1}}\left(1-\mathrm{e}^{-(\alpha+\mathrm{r}) \Delta_{1}}\right)$

$+c_{s} e^{-r\left(t_{r}+\Delta_{1}+\Delta_{2}\right)} e^{-(b+\alpha) \Delta_{1}}\left[\frac{\alpha+r}{r}-e^{b t_{0}+r \Delta_{2}}\right.$

$$
+\frac{r}{r-b}\left(e^{b t_{0}+r \Delta_{2}}-e^{b t_{s}}\right)
$$

$e^{-(b+\alpha) \Delta_{1}}-\left(\frac{b+\alpha}{r-b}\right) e^{b t_{0}+r \Delta_{2}}$

$\left.\left(1-\mathrm{e}^{-(\mathrm{b}+\alpha) \Delta_{1}}\right)-\left(\frac{\mathrm{b}+\alpha}{\mathrm{r}}\right) \mathrm{e}^{-(\mathrm{b}+\alpha) \Delta_{1}}\right]$

$\left.-\mathrm{c}_{\mathrm{s}} \mathrm{e}^{-\mathrm{r}\left(\mathrm{t}_{\mathrm{r}}+\Delta_{1}+\Delta_{2}\right)} \mathrm{r} \Delta_{2} \mathrm{e}^{-2(\mathrm{~b}+\alpha) \Delta_{1}}\right\} /\left(\mathrm{t}_{\mathrm{r}}+\Delta_{1}+\Delta_{2}\right)$

$\frac{\partial^{2} \mathrm{Tc}_{1}}{\partial \Delta_{2} \partial \mathrm{t}_{\mathrm{r}}}=\left(-\mathrm{c}_{\mathrm{s}} \mathrm{a}\left\{\mathrm{e}^{-\mathrm{r}\left(\mathrm{t}_{\mathrm{r}}+\Delta_{1}+\Delta_{2}\right)}\left(\frac{\mathrm{r}}{\mathrm{r}-\mathrm{b}} \mathrm{e}^{\mathrm{bt} \mathrm{t}_{0}+\mathrm{r} \Delta_{2}}-1\right)\right.\right.$

$\left.-\left[\frac{1}{r-b}\left(e^{b t_{0}+r \Delta_{2}}-e^{b t_{s}}\right)-\Delta_{2}\right] r e^{-r\left(t_{r}+\Delta_{1}+\Delta_{2}\right)}\right\}$

$$
\left.\left(1+\frac{\mathrm{d} \Delta_{1}}{\mathrm{dt}_{\mathrm{r}}}\right)-\frac{\partial \mathrm{Tc}_{1}}{\partial \mathrm{t}_{\mathrm{r}}}\right) /\left(\mathrm{t}_{\mathrm{r}}+\Delta_{1}+\Delta_{2}\right)
$$

$\frac{\partial^{2} \mathrm{Tc}_{1}}{\partial \Delta_{2} \partial \mathrm{t}_{\mathrm{r}}}=\mathrm{c}_{\mathrm{s}} \mathrm{a} \mathrm{e}^{-\mathrm{r}\left(\mathrm{t}_{\mathrm{r}}+\Delta_{1}+\Delta_{2}\right)}\left(1-\frac{\mathrm{r}}{\mathrm{r}-\mathrm{b}} \mathrm{e}^{\mathrm{bt}} \mathrm{r} \Delta_{2}\right)$

$$
\left(1+\frac{\mathrm{d} \Delta_{1}}{\mathrm{dt}_{\mathrm{r}}}\right) /\left(\mathrm{t}_{\mathrm{r}}+\Delta_{1}+\Delta_{2}\right)
$$

$$
\frac{\partial^{2} \mathrm{Tc}_{1}}{\partial \mathrm{t}_{\mathrm{r}} \partial \Delta_{2}}=\mathrm{c}_{\mathrm{s}} \mathrm{a}\left[-\mathrm{r}\left(1+\frac{\mathrm{d} \Delta_{1}}{\mathrm{dt}_{\mathrm{r}}}\right) \mathrm{e}^{-\mathrm{r}\left(\mathrm{t}_{\mathrm{r}}+\Delta_{1}+\Delta_{2}\right)}\right.
$$

$$
\begin{aligned}
& \left.\left(\frac{\mathrm{e}^{\mathrm{bt}}}{\mathrm{r}-\mathrm{b}}+\Delta_{2}-\frac{1}{\mathrm{r}}\right)\right] /\left(\mathrm{t}_{\mathrm{r}}+\Delta_{1}+\Delta_{2}\right) \\
& \frac{\partial^{2} \mathrm{Tc}_{1}}{\partial \mathrm{t}_{\mathrm{r}} \partial \Delta_{2}}=\mathrm{c}_{\mathrm{s}} \mathrm{ae}^{-\mathrm{r}\left(\mathrm{t}_{\mathrm{r}}+\Delta_{1}+\Delta_{2}\right)}\left(1-\mathrm{r} \Delta_{2}-\frac{\mathrm{r}}{\mathrm{r}-\mathrm{b}} \mathrm{e}^{\mathrm{b \textrm {t } _ { \mathrm { s } }}}\right) \\
& \left(1+\frac{\mathrm{d} \Delta_{1}}{\mathrm{dt}}\right) /\left(\mathrm{t}_{\mathrm{r}}+\Delta_{1}+\Delta_{2}\right) \\
& \mathrm{And}^{33)} \\
& \frac{\partial^{2} \mathrm{Tc}_{1}}{\partial \Delta_{2}^{2}}=\left\{\mathrm { c } _ { \mathrm { s } } \mathrm { a } \left[\mathrm{e}^{-\mathrm{r}\left(\mathrm{t}_{\mathrm{r}}+\Delta_{1}+\Delta_{2}\right)}(-\mathrm{r})\left(\frac{1}{\mathrm{r}-\mathrm{b}} \mathrm{e}^{\mathrm{bt} \mathrm{s}_{\mathrm{s}}}+\Delta_{2}-\frac{1}{\mathrm{r}}\right)\right.\right. \\
& \left.\left.+\mathrm{e}^{-\mathrm{r}\left(\mathrm{t}_{\mathrm{r}}+\Delta_{1}+\Delta_{2}\right)}\right]-\frac{2 \partial \mathrm{Tc} \mathrm{c}_{1}}{\partial \Delta_{2}}\right\} /\left(\mathrm{t}_{\mathrm{r}}+\Delta_{1}+\Delta_{2}\right) \\
& \frac{\partial^{2} \mathrm{Tc}_{1}}{\partial \Delta_{2}^{2}}=\mathrm{e}^{-\mathrm{r}\left(\mathrm{t}_{\mathrm{r}}+\Delta_{1}+\Delta_{2}\right)} \mathrm{c}_{\mathrm{s}} \mathrm{a}\left[\frac{-\mathrm{r}}{\mathrm{r}-\mathrm{b}} \mathrm{e}^{\mathrm{bt}}-\mathrm{r} \Delta_{2}+2\right] /\left(\mathrm{t}_{\mathrm{r}}+\Delta_{1}+\Delta_{2}\right)
\end{aligned}
$$

From (25)

$c_{s} e^{-r\left(t_{r}+\Delta_{1}+\Delta_{2}\right)}\left(\frac{1}{r-b} e^{b t_{0}+r \Delta_{2}}-\frac{1}{r}\right) e^{-(b+\alpha) \Delta_{1}}$ $=\frac{c h_{2}+\beta c_{p}}{\beta+r}\left(e^{(b+\beta) t_{r}}-e^{(b-r) t_{r}}\right)$

$+\frac{\mathrm{ch}_{1}+\alpha c_{\mathrm{p}}}{\alpha+\mathrm{r}} \mathrm{e}^{(\mathrm{b}-\mathrm{r}) \mathrm{t}_{\mathrm{r}}}\left(1-\mathrm{e}^{-(\alpha+\mathrm{r}) \Delta_{1}}\right)$

$=c_{s} e^{-r\left(t_{r}+\Delta_{1}+\Delta_{2}\right)}\left(\frac{1}{r-b} e^{b t_{0}+r \Delta_{2}}-\frac{1}{r}\right) e^{-(b+\alpha) \Delta_{1}}$ $=\frac{c h_{2}+\beta c_{p}}{\alpha+r}\left[\frac{\alpha+r}{\beta+r}\left(e^{(b+\beta) t_{r}}-e^{(b-r) t_{r}}\right)\right.$

$\left.+\frac{\mathrm{ch}_{1}+\alpha \mathrm{c}_{\mathrm{p}}}{\mathrm{ch}_{2}+\beta \mathrm{c}_{\mathrm{p}}} \mathrm{e}^{(\mathrm{b}-\mathrm{r}) \mathrm{t}_{\mathrm{r}}}\left(1-\mathrm{e}^{-(\alpha+\mathrm{r}) \Delta_{1}}\right)\right]$

$=c_{S} e^{-r\left(t_{r}+\Delta_{1}+\Delta_{2}\right)}\left(\frac{1}{r-b} e^{b t_{0}+r \Delta_{2}}-\frac{1}{r}\right) e^{-(b+\alpha) \Delta_{1}}$ 
Published Online July 2019 in IJEAST (http://www.ijeast.com)

$<\frac{c h_{2}+\beta c_{p}}{\alpha+r}\left(e^{(b+\beta) t_{r}}-e^{(b-r) t_{r}}+e^{(b-r) t_{r}}-e^{(b-r) t_{r}} e^{-(\alpha+r) \Delta_{1}}\right)$

$<\frac{\mathrm{ch}_{2}+\beta \mathrm{c}_{\mathrm{p}}}{\alpha+\mathrm{r}}\left(\mathrm{e}^{(\mathrm{b}+\beta) \mathrm{t}_{\mathrm{r}}}-\mathrm{e}^{(\mathrm{b}-\mathrm{r}) \mathrm{t}_{\mathrm{r}}} \mathrm{e}^{-(\alpha+\mathrm{r}) \Delta_{1}}\right)$

$$
\left(\text { by } \frac{\alpha+\mathrm{r}}{\beta+\mathrm{r}}<1 \text { and } \frac{\mathrm{ch}_{1}+\alpha \mathrm{c}_{\mathrm{p}}}{\mathrm{ch}_{2}+\beta \mathrm{c}_{\mathrm{p}}}<1\right)
$$

Thus from (31) it is obvious that

$\frac{\partial^{2} \mathrm{Tc}_{1}}{\partial \mathrm{t}_{\mathrm{r}}^{2}}=\mathrm{a}\left\{\left(\mathrm{ch}_{2}+\beta \mathrm{c}_{\mathrm{p}}\right) \mathrm{e}^{(\mathrm{b}+\beta) \mathrm{t}_{\mathrm{r}}}-\left(\mathrm{ch}_{1}+\alpha \mathrm{c}_{\mathrm{p}}\right)-\mathrm{e}^{-(\alpha+\mathrm{r}) \Delta_{1}}\left(1-\mathrm{e}^{-(\alpha+\mathrm{r}) \Delta_{1}}\right)\right.$

$+c_{s} e^{-r\left(t_{r}+\Delta_{1}+\Delta_{2}\right)} e^{-(b+\alpha) \Delta_{1}}\left[\frac{\alpha+r}{r}-e^{b t_{0}+r \Delta_{2}}\right.$

$+\frac{r}{r-b}\left(e^{b t_{0}+r \Delta_{2}}-e^{b t_{s}}\right) e^{-(b+\alpha) \Delta_{1}}$

$-\left(\frac{\mathrm{b}+\alpha}{\mathrm{r}-\mathrm{b}}\right) \mathrm{e}^{\mathrm{bt} \mathrm{t}_{0}+\mathrm{r} \Delta_{2}}\left(1-\mathrm{e}^{-(\mathrm{b}+\alpha) \Delta_{1}}\right)$

$\left.-\frac{\mathrm{b}+\alpha}{\mathrm{r}} \mathrm{e}^{-(\mathrm{b}+\alpha) \Delta_{1}}\right]$

$\left.-\mathrm{c}_{\mathrm{s}} \mathrm{e}^{-\mathrm{r}\left(\mathrm{t}_{\mathrm{r}}+\Delta_{1}+\Delta_{2}\right)} \mathrm{r} \Delta_{2} \mathrm{e}^{-2(\mathrm{~b}+\alpha) \Delta_{1}}\right\} /\left(\mathrm{t}_{\mathrm{r}}+\Delta_{1}+\Delta_{2}\right)>0$

Therefore,

$\frac{\partial^{2} \mathrm{Tc}_{1}}{\partial \mathrm{t}_{\mathrm{r}}^{2}} \cdot \frac{\partial^{2} \mathrm{Tc}_{1}}{\partial \Delta_{2}^{2}}-\frac{\partial^{2} \mathrm{Tc}_{1}}{\partial \Delta_{2} \partial \mathrm{t}_{\mathrm{r}}} \cdot \frac{\partial^{2} \mathrm{Tc}_{1}}{\partial \mathrm{t}_{\mathrm{r}} \partial \Delta_{2}}$

$=\mathrm{a}^{2}\left\{\left(\mathrm{ch}_{2}+\beta \mathrm{c}_{\mathrm{p}}\right) \mathrm{e}^{(\mathrm{b}+\beta) \mathrm{t}_{\mathrm{r}}}-\left(\mathrm{ch}_{1}+\alpha \mathrm{c}_{\mathrm{p}}\right) \mathrm{e}^{-(\alpha+\mathrm{r}) \Delta_{1}}\left(1-\mathrm{e}^{-(\alpha+\mathrm{r}) \Delta_{1}}\right)\right.$

$+\mathrm{c}_{\mathrm{s}} \mathrm{e}^{-\mathrm{r}\left(\mathrm{t}_{\mathrm{r}}+\Delta_{1}+\Delta_{2}\right)} \mathrm{e}^{-(\mathrm{b}+\alpha) \Delta_{1}}\left[\frac{\alpha+\mathrm{r}}{\mathrm{r}}-\mathrm{e}^{\mathrm{bt}_{0}+\mathrm{r} \Delta_{2}}\right.$

$$
+\frac{r}{r-b}\left(e^{b t_{0}+r \Delta_{2}}-e^{b t_{s}}\right) e^{-(b+\alpha) \Delta_{1}}-\frac{b+\alpha}{r-b} e^{b t_{0}+r \Delta_{2}}\left(1-e^{-(b+\alpha) \Delta_{1}}\right)
$$$$
\left.\left.-\frac{\mathrm{b}+\alpha}{\mathrm{r}} \mathrm{e}^{-(\mathrm{b}+\alpha) \Delta_{1}}\right]-\mathrm{c}_{\mathrm{s}} \mathrm{e}^{-\mathrm{r}\left(\mathrm{t}_{\mathrm{r}}+\Delta_{1}+\Delta_{2}\right)} \mathrm{r} \Delta_{2} \mathrm{e}^{-2(\mathrm{~b}+\alpha) \Delta_{1}}\right\}
$$$$
c_{s} e^{-r\left(t_{r}+\Delta_{1}+\Delta_{2}\right)}\left(-\frac{r}{r-b} e^{b t_{s}}-r \Delta_{2}+2\right) /\left(t_{r}+\Delta_{1}+\Delta_{2}\right)
$$

$$
\left[-c_{s} e^{-r\left(t_{r}+\Delta_{1}+\Delta_{2}\right)}\left(1-r \Delta_{2}-\frac{r}{r-b} e^{b t_{s}}\right)\right.
$$

$$
\left.\left(1+\frac{\mathrm{d} \Delta_{1}}{\mathrm{dt}_{\mathrm{r}}}\right) /\left(\mathrm{t}_{\mathrm{r}}+\Delta_{1}+\Delta_{2}\right)\right]^{2}
$$

$=\left[\mathrm{a}^{2} \mathrm{c}_{\mathrm{s}} \mathrm{e}^{-\mathrm{r}\left(\mathrm{t}_{\mathrm{r}}+\Delta_{1}+\Delta_{2}\right)}\left\{\left(\mathrm{ch}_{2}+\beta \mathrm{c}_{\mathrm{p}}\right) \mathrm{e}^{(\mathrm{b}+\beta) \mathrm{t}_{\mathrm{r}}}-\left(\mathrm{ch}_{1}+\alpha \mathrm{c}_{\mathrm{p}}\right) \mathrm{e}^{-(\alpha+\mathrm{r}) \Delta_{1}}\right.\right.$

$\left(1-\mathrm{e}^{-(\alpha+\mathrm{r}) \Delta_{1}}\right)$
$+\mathrm{c}_{\mathrm{s}} \mathrm{e}^{-\mathrm{r}\left(\mathrm{t}_{\mathrm{r}}+\Delta_{1}+\Delta_{2}\right)} \mathrm{e}^{-(\mathrm{b}+\alpha) \Delta_{1}}\left[\frac{\alpha+\mathrm{r}}{\mathrm{r}}-\mathrm{e}^{\mathrm{bt}_{0}+\mathrm{r} \Delta_{2}}\right.$

$+\frac{r}{r-b}\left(e^{b t_{0}+r \Delta_{2}}-e^{b t_{s}}\right) e^{-(b+\alpha) \Delta_{1}}-\frac{b+\alpha}{r-b} e^{b t_{0}+r \Delta_{2}}\left(1-e^{-(b+\alpha) \Delta_{1}}\right)$

$$
\left.\begin{array}{r}
-\frac{b+\alpha}{r} e^{-(b+\alpha) \Delta_{1}} \\
-c_{s} e^{-r\left(t_{r}+\Delta_{1}+\Delta_{2}\right)} r \Delta_{2} e^{-2(b+\alpha) \Delta_{1}}
\end{array}\right]
$$

$$
\left(-\frac{r}{r-b}-r \Delta_{2}+2\right)-c_{s} e^{-r\left(t_{r}+\Delta_{1}+\Delta_{2}\right)}\left(1-r \Delta_{2}-\frac{r}{r-b} e^{b t_{s}}\right)
$$

$$
\left.\mathrm{e}^{-2(\mathrm{~b}+\alpha) \Delta_{1}}\right] /\left(\mathrm{t}_{\mathrm{r}}+\Delta_{1}+\Delta_{2}\right)^{2}>0 \text {. }
$$

As a result, the solution that satisfies

(23) and (24) minimizes $\mathrm{Tc}_{1}$ in (18). 
In this paper, we propose a model for deteriorating items the optimal replenishment cycle for the two-warehouse inventory problem under inflation, in which the inventory deteriorates at a constant rate over time and shortages are allowed with exponential rate of demand. The two-warehouse inventory model for deteriorating items with exponential demand rate and shortages under inflation is considered for this model, the optimal solution obtained. The proposed model can be extended in numerous ways. For example, we may extend the exponential demand to a more generalized demand pattern that fluctuates with time. Also, we may consider the unit purchase cost, the inventory holding cost, and others are also fluctuating with time. Finally, we may generalize the model to allow for quantity discount.

\section{REFERENCES:}

- Buzacott, J.A. (1975):Economic order quantities with inflation.

Operational Research Quarterly, 26, 3, 553 558 .

- Bierman, H. and Thomas, J. (1977):Inventory decisions under inflationary conditions. Decision Sciences, $8,151-155$.

- Bose, S., Goswami, A. and Chaudhuri, K.S. (1995):An EOQ model for deteriorating items with linear time dependent demand rate and shortages under inflation and time discounting. Journal of the Operational Research Society, 46, 6, 771-782.

- Teng, J.T. and Yang, H.L. (2004): Deterministic economic order quantity models with partial backlogging when demand and cost are fluctuating with time. Journal of the Operational Research Society, 55(5), 495-503.

- Pal, A.K., Bhunia, A.K. and Mukherjee, R.N. (2006): Optimal lot size model for deteriorating items with demand rate dependent on displayed stock level (DSL) and partial backordering. European Journal of Operational Research, 175(2), 977-991.

- Singh, C. and Singh, S.R. (2010) 'Supply chain model with stochastic lead time under imprecise partially backlogging and fuzzy ramp-type demand for expiring items', Int. J. Operational Research, Vol. 8, No. 4, pp.511-522.

- Skouri, K., Konstantaras, I., Papachristos, S., \& Teng, J.T. (2011). Supply chain models for deteriorating products with ramp type demand rate under permissible delay in payments. Expert Systems with Applications, 38, $14861-14869$.

- Singh, S.R. and Saxena, N. (2012) 'An optimal returned policy for a reverse logistics inventory model with backorders', Advances in Decision Sciences, Article ID 386598, $21 \mathrm{pp}$

- Zang J, Bai Z, Tang W. Optimal pricing policy for deteriorating items with preservation technology investment. J Ind Manag Optim. 2014;10(4):2014.

- H. Nagar and P. Surana (2015) Fuzzy Inventory Model for Deteriorating Items with Fluctuating Demand and Using Inventory Parameters as Pentagonal Fuzzy Numbers, Journal of Computer and Mathematical Sciences, Vol. 6(2), 55-66

- Banu A, Mondal SK. Analysis of credit linked demand in an inventory model with varying ordering cost. Springerplus. 2016;5:926. doi: 10.1186/s40064016-2567-9. 